\title{
Isomorphism questions for metric ultraproducts of finite quasisimple groups
}

\author{
Jakob Schneider \\ Communicated by John S. Wilson
}

\begin{abstract}
New results on metric ultraproducts of finite simple groups are established. We show that the isomorphism type of a simple metric ultraproduct of groups $X_{n_{i}}(q)$ $(i \in I)$ for $X \in\left\{\mathrm{PGL}, \mathrm{PSp}, \mathrm{PGO}^{(\varepsilon)}\right.$, PGU $(\varepsilon= \pm)$ along an ultrafilter $\mathcal{U}$ on the index set $I$ for which $n_{i} \rightarrow u \infty$ determines the type $X$ and the field size $q$ up to the possible isomorphism of a metric ultraproduct of groups $\operatorname{PSp}_{n_{i}}(q)$ and a metric ultraproduct of groups $\mathrm{PGO}_{n_{i}}^{(\varepsilon)}(q)$. This extends results of [A. Thom and J. Wilson, Metric ultraproducts of finite simple groups, Comp. Rend. Math. 352 (2014), no. 6, 463-466].
\end{abstract}

\section{Introduction}

In $[5,6]$, Thom and Wilson discussed various properties of metric ultraproducts of finite simple groups. In particular, they asked which such ultraproducts can be isomorphic. In [5, Theorem 2.2], a metric ultraproduct of alternating groups is distinguished from a metric ultraproduct of classical groups of Lie type, where the permutation degrees resp. dimensions of the natural module tend to infinity. This is done by considering the structure of centralizers of torsion elements in these groups (see [5, Theorems 2.8 and 2.9]). In the case of a metric ultraproduct of classical groups of Lie type, in [5, Theorem 2.8], investigating the structure of such centralizers of semisimple and unipotent torsion elements, Thom and Wilson even extract the "limit characteristic" of the group. At the end of [5, Section 2], they ask which metric ultraproducts of classical groups of different types can be isomorphic.

In this note, we will give an almost complete answer to this question in the case when the field sizes are bounded. We will show that, for a metric ultraproduct of alternating or classical groups of Lie type of unbounded rank over fields of bounded size, one can extract the Lie type (up to one exception). Also one can extract the "limit field size". Our results are summed up in Theorem 1 below. To state it, we first need to introduce some notation which we fix for the whole article. 
Let $\mathscr{H}=\left(H_{i}\right)_{i \in I}$ be a sequence of groups where either $H_{i}=\mathrm{S}_{n_{i}}$ is a symmetric group or $H_{i}=X_{i}\left(q_{i}\right)$ is a classical group of Lie type $X_{i}$ over the finite field $\mathbb{F}_{q_{i}}$ with $q_{i}$ elements (resp. $\mathbb{F}_{q_{i}^{2}}$ in the unitary case, $i \in I$ ). In the latter case, we let each $X_{i}$ be one of $\mathrm{GL}_{n_{i}}, \mathrm{Sp}_{2 m_{i}}, \mathrm{GO}_{2 m_{i}}^{ \pm}, \mathrm{GO}_{2 m_{i}+1}\left(q_{i}\right.$ odd), or $\mathrm{GU}_{n_{i}}$ for suitable $m_{i}, n_{i} \in \mathbb{Z}_{+}(i \in I)$.

Recall that a norm $\ell$ on a group $H$ is a function $H \rightarrow[0, \infty]$ such that $\ell(h)=0$ iff $h=1_{H}, \ell(h)=\ell\left(h^{-1}\right)=\ell\left(h^{g}\right)$, and $\ell(g h) \leq \ell(g)+\ell(h)$ for all $g, h \in H$. Call a pair $(H, \ell)$, where $H$ is a group and $\ell$ a norm on it, a normed group. Recall that the metric ultraproduct of a sequence of normed groups $\left(H_{i}, \ell_{i}\right)_{i \in I}$ along an ultrafilter $\mathcal{U}$ on the set $I$ is defined as the quotient $\prod_{i \in I} H_{i} / N$, where $N:=\left\{\left(h_{i}\right)_{i \in I} \in \prod_{i \in I} H_{i} \mid \lim \mathcal{U} \ell_{i}\left(h_{i}\right)=0\right\}$ is the normal subgroup of $\mathcal{U}$-null sequences.

Throughout, let $G:=\mathscr{H}_{\mathcal{U}}^{\text {met }}$ be the metric ultraproduct of the groups $H_{i}$ from above equipped with the normalized Hamming norm

$$
\ell_{\mathrm{H}}(\sigma):=|\operatorname{supp}(\sigma)| / n=|\{x \in\{1, \ldots, n\} \mid x . \sigma \neq x\}| / n
$$

resp. the normalized rank norm $\ell_{\mathrm{rk}}(g):=\operatorname{rk}(1-g) / n$ when $H_{i}$ is a symmetric resp. a classical linear group of Lie type along the ultrafilter $U$. Assume that the permutation degrees resp. dimensions of the natural module $n_{i}$ of $H_{i}(i \in I)$ tend to infinity along $\mathcal{U}$.

Note that, since $\mathcal{U}$ is an ultrafilter, we may assume that each group $H_{i}$ is of the same type, i.e., all groups $H_{i}$ are either symmetric, linear, symplectic, orthogonal, or unitary groups. In these five distinct cases, we write $\mathrm{S}_{u}, \mathrm{GL}_{\mathcal{u}}, \mathrm{Sp}_{u}, \mathrm{GO}_{u}$, or $\mathrm{GU}_{u}$ for $G$. Also, when the field sizes $q_{i}$ are bounded, we may assume that $q_{i}=q$ is constant $(i \in I)$, setting $q:=\lim u q_{i}$. Throughout the article, set $Z:=\mathbf{Z}(G)$ to be the center of $G$ and $\bar{G}:=G / Z$.

If the groups $H_{i}(i \in I)$ are symmetric groups, then $Z=\mathbf{1}$ and $\bar{G}=G$. Now assume that all groups $H_{i}$ are of type $X\left(q_{i}\right)(i \in I$; i.e., they are not symmetric groups). Then $\bar{G}=G / Z=\overline{\mathscr{H}}_{\mathcal{U}}^{\text {met }}$ is the metric ultraproduct of the groups $\bar{H}_{i}:=H_{i} / \mathbf{Z}\left(H_{i}\right)$ with respect to the projective rank norm

$$
\ell_{\mathrm{pr}}(\bar{h}):=\inf \left\{\ell_{\mathrm{rk}}(h) \mid h \text { is a lift of } \bar{h} \text { in } H\right\},
$$

which is defined on the general projective linear group. By the results from [3], $\bar{G}$ is the unique simple quotient of $G$.

Similarly to the above, write PGL $u, \mathrm{PSp}_{u}, \mathrm{PGO}_{u}$, or $\mathrm{PGU}_{u}$ for $\bar{G}$ when all the groups $H_{i}(i \in I)$ are linear, symplectic, orthogonal, or unitary groups. Moreover, in this case, if all the fields $\mathbb{F}_{q_{i}}(i \in I)$ are equal to $\mathbb{F}_{q}$ (or $\mathbb{F}_{q^{2}}$ in the unitary case), we write $\mathrm{GL}_{u}(q), \mathrm{Sp}_{u}(q), \mathrm{GO}_{u}(q), \mathrm{GU}_{u}(q)$ resp. $\operatorname{PGL} u(q), \operatorname{PSp}_{u}(q)$, $\operatorname{PGO} u(q), \operatorname{PGU}_{u}(q)$ for $G$ resp. $\bar{G}$. Write GLu $(k)$ resp. PGLu $(k)$ for the metric 
ultraproduct of groups $\mathrm{GL}_{n_{i}}(k)$ resp. $\mathrm{PGL}_{n_{i}}(k)$ over the field $k(i \in I)$ along $U$. Write $\mathbf{M}_{n}(k)$ for the matrix ring of degree $n$ over the field $k$ and $\mathbf{P M}_{n}(k)$ for the associated projective space $\left(\mathbf{M}_{n}(k) \backslash\{0\}\right) / k^{\times}$. Set $\mathbf{M}_{n}(q):=\mathbf{M}_{n}\left(\mathbb{F}_{q}\right)$ and $\operatorname{PM}_{n}(q):=\mathbf{P M}_{n}\left(\mathbb{F}_{q}\right)$. Also write $\mathbf{M}_{\mathcal{U}}, \mathbf{M}_{\mathcal{U}}(q)$ resp. $\mathbf{P M}_{\mathcal{U}}, \mathbf{P M}_{u}(q)$ for the metric ultraproduct of the spaces $\mathbf{M}_{n_{i}}\left(q_{i}\right), \mathbf{M}_{n_{i}}(q)$ resp. $\mathbf{P M}_{n_{i}}\left(q_{i}\right), \mathbf{P M}_{n_{i}}(q)$ with respect to the metrics

$$
\begin{aligned}
& d_{\mathrm{rk}}(g, h):=\operatorname{rk}(g-h) / n, \\
& d_{\mathrm{pr}}(\bar{g}, \bar{h}):=\inf \left\{d_{\mathrm{rk}}(g, h) \mid g, h \text { are lifts of } \bar{g}, \bar{h}\right\} \quad(i \in I)
\end{aligned}
$$

so that $\mathrm{GL} u \subseteq \mathbf{M}_{u}, \mathrm{GL} u(q) \subseteq \mathbf{M}_{u}(q), \operatorname{PGL}_{u} \subseteq \mathrm{PM}_{u}, \operatorname{PGL} u(q) \subseteq \operatorname{PM}_{u}(q)$.

The main result of this article is now as follows.

Theorem 1. Let $\bar{G} \cong \bar{G}_{1} \cong \bar{G}_{2}$ with $G_{j}=X_{j} u_{j}\left(q_{j}\right)$, where

$$
X_{j} \in\{\mathrm{GL}, \mathrm{Sp}, \mathrm{GO}, \mathrm{GU}\} \quad(j=1,2) .
$$

Then it holds that $q_{1}=q_{2}$. Also we must have $X_{1}=X_{2}$ or $\left\{X_{1}, X_{2}\right\}=\{\mathrm{Sp}, \mathrm{GO}\}$. Moreover, an ultraproduct $\bar{X}_{1} u_{1}$ where the sizes $q_{i}$ of the finite fields $\mathbb{F}_{q_{i}}\left(i \in I_{1}\right)$ tend to infinity along $U_{1}$ cannot be isomorphic to an ultraproduct $\bar{X}_{2} u_{2}(q)$.

Let us conclude this introduction by saying some words about the proof of Theorem 1. Our strategy is to compute double centralizers of semisimple torsion elements of a fixed order $o \in \mathbb{Z}_{+}$in the above metric ultraproducts. If the sizes $q_{i}$ $\left(i \in I\right.$ ) of the fields $\mathbb{F}_{q_{i}}$ are bounded, it turns out that these are always finite abelian groups. Then we consider the maximal possible exponent which such a double centralizer can have. It turns out that this data is enough to determine the limit field size $q$ and the Lie type (up to the exception mentioned in Theorem 1). If the field sizes $q_{i}(i \in I)$ tend to infinity, a double centralizer of such a torsion element of order $o>2$ is always infinite. This separates this case from the former one. Throughout the article, we will make frequent use of the exposition given in [4, Subsection 3.4.2, § 1]. This paragraph in the author's $\mathrm{PhD}$ thesis provides a concise presentation of the classification of the possible indecomposable blocks of elements from classical groups of Lie type stabilizing a form (which is, of course, well known). For the convenience of the reader, we will quote the facts needed from it in the subsequent section.

\section{Notation and auxiliary results from [4]}

In this section, we introduce the necessary notation and quote the facts from [4] which we need for the proof of Theorem 1. Keep the notation from the introduction. Subsequently, $G$ will be one of $\mathrm{S} u, \operatorname{GLu}(q), \mathrm{Sp}_{u}(q), \mathrm{GO}_{u}(q)$, or $\operatorname{GU} u(q)$ 
(so that $\bar{G}$ is either $\mathrm{S}_{u}, \mathrm{PGL}_{u}(q), \operatorname{PSp}_{u}(q)$, $\operatorname{PGO}_{u}(q)$, or $\operatorname{PGU}_{u}(q)$, respectively). We refer to these five distinct cases as the symmetric case, the linear case, the symplectic case, the orthogonal case, and the unitary case, respectively. The last four cases are grouped together as the classical case. The symplectic and orthogonal case are grouped together as the bilinear case.

For the rest of this section, we assume that we are in the classical case. Then set $k=\mathbb{F}_{q}$ in the non-unitary case and $k=\mathbb{F}_{q^{2}}$ in the unitary case. Write $p:=\operatorname{char}(k)$ for the characteristic of the field $k$.

Subsequently, assume that we are not in the linear case. Then we let $\sigma: k \rightarrow k$ be the $q$-Frobenius map $x \mapsto x^{q}$, i.e., $\sigma=\mathrm{id}_{k}$ in the bilinear case, whereas $\sigma$ is the unique involution of $k=\mathbb{F}_{q^{2}}$ in the unitary case. For a monic polynomial

$$
\chi=a_{0}+a_{1} X+\cdots+a_{k-1} X^{k-1}+X^{k} \in k[X],
$$

write $\chi^{*}=a_{0}^{-\sigma} X^{\operatorname{deg}(\chi)} \chi^{\sigma}\left(X^{-1}\right)$ for the dual polynomial of $\chi$. Here

$$
\chi^{\sigma}=a_{0}^{\sigma}+a_{1}^{\sigma} X+\cdots+a_{k-1}^{\sigma} X^{k-1}+X^{k}
$$

is the polynomial $\chi$ with coefficients twisted by the automorphism $\sigma$. Call $\chi$ selfdual iff $\chi=\chi^{*}$. Furthermore, write $f_{i}$ for the sesquilinear forms stabilized by the group $H_{i}$ (and $Q_{i}$ for the quadratic forms in the orthogonal case for $p=2$, $i \in I$ ). Note that a metric ultraproduct of groups $\mathrm{GO}_{2 m_{i}+1}(q)$ for $q$ a power of $p=2$ with respect to the projective rank norm where the $m_{i}$ tend to infinity along the chosen ultrafilter is isomorphic to such an ultraproduct of groups $\operatorname{Sp}_{2 m_{i}}(q)$. Actually, $\mathrm{GO}_{2 m_{i}+1}(q) \cong \operatorname{Sp}_{2 m_{i}}(q)$, but the natural module of $\mathrm{GO}_{2 m_{i}+1}(q)$ has one-dimensional radical $\operatorname{rad}\left(f_{i}\right)$, whereas $\operatorname{rad}\left(f_{i}\right)=0$ in the second case. Hence we may and do assume that the forms $f_{i}(i \in I)$ are all non-singular and exclude groups of type $\mathrm{GO}_{2 m+1}(q)$ for $q$ even. Write

$$
F(\chi):=\left(\begin{array}{ccccc}
0 & 1 & 0 & \cdots & 0 \\
\vdots & \ddots & \ddots & \ddots & \vdots \\
\vdots & & \ddots & \ddots & 0 \\
0 & \cdots & \cdots & 0 & 1 \\
-a_{0} & \cdots & \cdots & \cdots & -a_{k-1}
\end{array}\right)
$$

for the Frobenius block associated to the monic polynomial

$$
\chi=a_{0}+a_{1} X+\cdots+a_{k-1} X^{k-1}+X^{k} \in k[X],
$$

i.e., multiplication by $\bar{X}$ in the quotient ring $k[X] /(\chi)$ represented in the basis

$$
1, \bar{X}, \ldots, \bar{X}^{k-1} \text {. }
$$


Recall that, by the existence of the generalized Jordan normal (or primary rational canonical form) form, each linear transformation $g \in \mathbf{M}_{n}(k)$ can be written as

$$
g \cong \bigoplus_{\substack{\chi \in k[X] \\ \chi \text { monic primary }}} F(\chi)^{\oplus c_{\chi}(g)},
$$

where the $c_{\chi}(g)$ are uniquely determined. A polynomial $\chi \in k[X]$ is here called primary iff it is the power $i^{e}\left(e \in \mathbb{Z}_{+}\right)$of an irreducible polynomial $i \in k[X]$.

Here are the results needed from [4]. Subsequently, the group $H$ is one of the groups $H_{i}(i \in I)$, and the form $f$ is alternating bilinear, symmetric bilinear, or $\sigma$-sesquilinear in the symplectic, orthogonal, and unitary case, respectively. Hence the sign $\varepsilon$ of $f$ is -1 in the symplectic case, and $\varepsilon=+1$ in the orthogonal and unitary case. The form $Q$ is a compatible quadratic form in the orthogonal case for $p=2$, i.e., $Q(u+v)=Q(u)+f(u, v)+Q(v)$ for all $u$, $v$. Recall that two sesquilinear forms $f$ and $f^{\prime}$ (or quadratic forms $Q$ and $Q^{\prime}$ ) on a vector space $V$ are called linearly equivalent if $f(u, v)=f^{\prime}(u \cdot g, v . g)\left(\right.$ resp. $\left.Q(v)=Q^{\prime}(v \cdot g)\right)$ for all $u, v \in V$ and some $g \in \operatorname{GL}(V)$.

Fact 1 ([4, Fact 3.40]). The following hold.

(i) Whenever $\chi \in k[X]$ is (monic) self-dual and is not divided by $X \pm 1$ in the bilinear case, then there exists a non-singular form $f$ (coming from a quadratic form $Q$ when $p=2$ ) which is preserved by $F(\chi)$ (and $Q$ as well). The form $f$ (and $Q$ ) is even unique up to linear equivalence.

(ii) Assume now that $\chi=(X \pm 1)^{e}$ (which is self-dual) and we are in the bilinear case.

(a) At first, assume that $p$ is odd. Then, when $\varepsilon=(-1)^{e-1}$, there is a unique non-singular form $f$ (up to linear equivalence) preserved by $F(\chi)$, and when $\varepsilon=(-1)^{e}$, there is a unique non-singular form $f$ (up to linear equivalence) preserved by $F(\chi)^{\oplus 2}$, but no non-singular form is preserved by $F(\chi)$.

(b) If $p=2$, we use [2, Theorem 3.1] to see that if $g \cong F(\chi)^{\oplus c}$, i.e., $g$ acts $\chi$-isotypically (see the beginning of Section 3), then any non-singular form $f$ (and the associated quadratic form $Q$ ) restricts to all but at most constantly many blocks $F(\chi)$ to the indecomposable $W(e)$ from this theorem (which acts as $F(\chi)^{\oplus 2}$ ).

An indecomposable block of an element $g$ from a classical group of Lie type stabilizing the sesquilinear form $f$ with natural module $V$ is a subspace $W \neq 0$ on which $f$ is non-singular (i.e., $W \cap W^{\perp}=0$ ) and which is minimal with this 
property. Out of the theory of indecomposables presented in [4, Subsection 3.4.2, $\S 1]$, one can also deduce the following fact which we will use in the text.

Fact 2. Let $H=\mathrm{GI}(V, f)$ (resp. $H=\mathrm{GI}(V, Q)$ in the orthogonal case for $p=2$ ) be a classical group of Lie type stabilizing the non-singular form $f$ (and $Q$ ). Let $g \in H$ and $h: U \rightarrow W$ be a partial isometry between the subspaces $U, W \leq V$ commuting with $g$. Then $h$ extends to a full isometry $h^{\prime} \in H$ which commutes with $g$.

The next result, which can also be deduced from [4, Subsection 3.4.2, §1], describes the shape of $f$ on a "semisimple" indecomposable block.

Fact 3. Let $H$ be as in Fact 2, and let $g \in H$ be a semisimple element, i.e., for each Frobenius block $F(\chi)$ in the generalized Jordan normal form of $g$ (see above), we have that $\chi=i^{1}$ is irreducible (or alternatively the order $\operatorname{ord}(h)$ of $h$ is coprime to the characteristic $p$ of the group $H$ ). Then the indecomposable blocks of $g$ look as follows. Assume $W \leq V$ is an indecomposable block of $g$ such that $\left.g\right|_{W}$ has a Jordan block $F(\chi)$ acting on the subspace $U \leq W$ of dimension $\operatorname{deg}(\chi)$ for $\chi \in k[X]$ irreducible. Write $K_{\chi}:=k[X] /(\chi)$, and let $\lambda_{\chi}:=\bar{X} \in K_{\chi}$ be a root of $\chi$ in $K_{\chi}$. Let $\tau: K_{\chi^{*}} \rightarrow K_{\chi}$ be the field isomorphism given by $\left.\tau\right|_{k}=\sigma$ and $\lambda_{\chi^{*}} \mapsto \lambda_{\chi}^{-1}$. If $\chi$ is self-dual, i.e., $\chi=\chi^{*}$, we clearly have that $\tau \in \operatorname{Aut}\left(K_{\chi} / k_{\sigma}\right)$ is an involution (or the identity in the bilinear case when $\chi=X \pm 1$ ).

(i) Assume $\chi \neq X \pm 1$ in the bilinear case.

(a) Assume $\chi$ is not self-dual. There exists $U^{\prime} \leq W$ such that $W=U \oplus U^{\prime}$ and $g$ acts as $F(\chi) \oplus F\left(\chi^{*}\right)$ on $U \oplus U^{\prime}$. So, as $k[g]$-modules, $U \cong K_{\chi}$ and $U^{\prime} \cong K_{\chi^{*}}$, where $g$ acts on $K_{\chi} \times K_{\chi^{*}} \cong U \oplus U^{\prime}=W$ as multiplication by $\left(\lambda_{\chi}, \lambda_{\chi^{*}}\right)$. The pairing $f$ on $W$ is given by

$$
U \times U^{\prime} \cong K_{\chi} \times K_{\chi^{*}} \ni(u, v) \mapsto \operatorname{tr}_{K_{\chi} / k}\left(u v^{\tau}\right),
$$

$\left.f\right|_{U}=0,\left.f\right|_{U^{\prime}}=0$, and extension to a sesquilinear form of sign $\varepsilon$ (this is clearly g-invariant). In the orthogonal case for $p=2$, the associated quadratic form $Q$ is uniquely determined by $f$ and $g$. It is given by $W=U \oplus U^{\prime} \ni u \oplus v \mapsto f(u, v)$.

(b) Assume now that $\chi$ is self-dual. Then $U=W$ and $g$ acts as $F(\chi)$ on $U$. Hence $U \cong K_{\chi}$ as a $k[g]$-module, where $g$ acts on $K_{\chi} \cong U$ as multiplication by $\lambda_{\chi}$. In this case, the form $f$ is given by

$$
U^{2} \cong K_{\chi}^{2} \ni(u, v) \mapsto \operatorname{tr}_{K_{\chi} / k}\left(\delta u v^{\tau}\right),
$$


where $\delta \in K_{\chi}$ is such that $\delta^{\tau}=\varepsilon \delta$. In the orthogonal case for $p=2$, the quadratic form $Q$ is given by $U \ni v \mapsto \operatorname{tr}_{\left(K_{\chi}\right)_{\tau} / k}\left(v v^{\tau}\right)$. Here $\left(K_{\chi}\right)_{\tau}$ denotes the fixed field of the involution $\tau$.

(ii) Assume now that $\chi=X \pm 1$ and we are in the bilinear case. Then we have $\left.g\right|_{W}=\mp \operatorname{id}_{W}$.

(a) If we are in the symplectic case, then $W \cong k^{2}=\mathbb{F}_{q}^{2}$ and $\left.f\right|_{W}$ is the unique (up to linear equivalence) non-singular alternating form on the vector space $W$.

(b) Assume we are in the orthogonal case and $p \neq 2$. Then $W \cong k=\mathbb{F}_{q}$ and $f$ is given by $(u, v) \mapsto \beta u v$, where $\beta \in \mathbb{F}_{q}^{\times}$is either 1 (the "standard square") or a "standard non-square".

(c) Assume we are in the orthogonal case and $p=2$. Then $W \cong k^{2}=\mathbb{F}_{q}^{2}$ and $f$ is the unique (up to linear equivalence) non-singular alternating form on it and $Q$ is a quadratic form of plus type or minus type, i.e., either $Q$ is given by $k^{2} \ni(u, v) \mapsto u v$ (plus type) or

$$
k^{2} \cong \mathbb{F}_{q^{2}} \ni v \mapsto \mathrm{N}_{\mathbb{F}_{q^{2}} / \mathbb{F}_{q}}(v)=v^{q+1},
$$

where $\mathrm{N}_{\mathbb{F}_{q^{2}} / \mathbb{F}_{q}}: \mathbb{F}_{q^{2}} \rightarrow \mathbb{F}_{q}$ is the field norm.

We will also make use of the relations presented in the next remark.

Remark 1. When $D$ is the block $W \cong k^{2}$ of case (ii) (b) of Fact 3 where $\beta=1$ and $E$ is the same block for a non-square $\beta \in \mathbb{F}_{q}^{\times}$, then $D \oplus D \cong E \oplus E$. Similarly, when $D$ is the block of minus type and $E$ the block of plus type on $W \cong k^{2}$ in Fact 3 (ii) (c), then $D \oplus D \cong E \oplus E$.

\section{Description of conjugacy classes in $\mathrm{S}_{u}, \mathrm{GL}_{u}(q)$, and PGL $u(q)$}

In this section, we give a description of the conjugacy classes of groups of type $\mathrm{S}_{u}$ or PGLu $(q)$. We will make use of this in the subsequent sections.

We start with some definitions. Write $S_{n}=\operatorname{Sym}(\underline{n})$ for the symmetric group acting on the set $\underline{n}:=\{1, \ldots, n\}$. Let $C_{k}(\sigma) \subseteq \mathrm{S}_{n}$ (for a permutation $\sigma \in \mathrm{S}_{n}$ and $\left.k \in \mathbb{Z}_{+}\right)$denote the set of all $k$-cycles of $\sigma$, and let $c_{k}(\sigma):=\left|C_{k}(\sigma)\right|$ denote the number of $k$-cycles of $\sigma$. Moreover, let $\Omega_{k}(\sigma)$ be the set on which all $k$-cycles of $\sigma$ act. Set $n_{k}(\sigma):=\left|\Omega_{k}(\sigma)\right|=k c_{k}(\sigma)$ to be the cardinality of $\Omega_{k}(\sigma)$. Call the permutation $\sigma \in \mathrm{S}_{n} k$-isotypic when $n_{k}(\sigma)=n$, i.e., $\sigma$ has only cycles of length $k$. Call $\sigma$ isotypic if it is $k$-isotypic for one number $k \in \mathbb{Z}_{+}$. Similarly, for $g \in \mathbf{M}_{n}(k)$ and a monic primary polynomial $\chi \in k[X]$, let $c_{\chi}(g)$ be the number 
of Frobenius blocks $F(\chi)$ in the generalized Jordan normal form of $g$ (see Section 2). Let $V_{\chi}(g)$ be the subspace on which $g$ acts as $F(\chi)^{\oplus c_{\chi}(g)}$ with respect to this normal form, and set $n_{\chi}(g):=\operatorname{dim}\left(V_{\chi}(g)\right)$. Clearly, $V_{\chi}(g)$ is not uniquely determined as it depends on the decomposition of the vector space $k^{n}$ associated to the chosen generalized Jordan normal form of $g$. However, by writing $V_{\chi}(g)$ we mean that these subspaces of $k^{n}$ are the decomposition of $k^{n}$ coming from such a normal form of $g$. Then, of course, $n_{\chi}(g)=\operatorname{deg}(\chi) c_{\chi}(g)$. For a monic polynomial $\chi \in k[X]$, say that $g$ is $\chi$-isotypic if $g \cong F(\chi)^{\oplus c}$ for some $c \in \mathbb{Z}_{+}$.

At first, we consider GL $u(q)$ instead of PGL $u(q)$. Subsequently, all polynomials from $k[X]$ that occur in the text are meant to be monic polynomials.

Conjugacy classes in $\mathrm{S}_{u}$ and $\mathrm{GL} u(q)$. For an integer $k \in \mathbb{Z}_{+}$and a polynomial $\xi \in k[X]$, define $r_{k}(\sigma):=\left|\operatorname{fix}\left(\sigma^{k}\right)\right| / n$ resp. $r_{\xi}(g):=\operatorname{dim}(\operatorname{ker}(\xi(g))) / n$ for $\sigma \in \mathrm{S}_{n}$ resp. $g \in \mathbf{M}_{n}(q)$. Here fix $(\sigma):=\{x \in \underline{n} \mid x . \sigma=x\}$ is the set of fixed points of the permutation $\sigma$. Extend this definition to $\mathrm{S} u$ and $\mathbf{M} u(q)$ by setting

$$
r_{k}(\sigma):=\lim _{u} r_{k}\left(\sigma_{i}\right) \text { and } \quad r_{\xi}(g):=\lim _{\mathcal{U}} r_{\xi}\left(g_{i}\right)
$$

for $\sigma={\overline{\left(\sigma_{i}\right)}}_{i \in I}$ resp. $g={\overline{\left(g_{i}\right)}}_{i \in I}$. Both expressions are well-defined since, for $\sigma={\overline{\left(\sigma_{i}\right)}}_{i \in I}={\overline{\left(\tau_{i}\right)}}_{i \in I} \in \mathrm{S} u$, one has

$$
\begin{aligned}
\frac{1}{n_{i}}|| \operatorname{fix}\left(\sigma_{i}^{k}\right)|-| \operatorname{fix}\left(\tau_{i}^{k}\right)|| & \leq d_{\mathrm{H}}\left(\sigma_{i}^{k}, \tau_{i}^{k}\right) \\
& \leq d_{\mathrm{H}}\left(\sigma_{i}^{k}, \sigma_{i}^{k-1} \tau_{i}\right)+\cdots+d_{\mathrm{H}}\left(\sigma_{i} \tau_{i}^{k-1}, \tau_{i}^{k}\right) \\
& =k d_{\mathrm{H}}\left(\sigma_{i}, \tau_{i}\right) \rightarrow u 0 .
\end{aligned}
$$

Similarly, if $g={\overline{\left(g_{i}\right)}}_{i \in I}={\overline{\left(h_{i}\right)}}_{i \in I} \in \operatorname{GL} u(q)$ and

$$
\xi=a_{0}+a_{1} X+\cdots+a_{k-1} X^{k-1}+X^{k} \in k[X],
$$

we have

$$
\begin{aligned}
& \frac{1}{n_{i}}\left|\operatorname{dim}\left(\operatorname{ker} \xi\left(g_{i}\right)\right)-\operatorname{dim}\left(\operatorname{ker} \xi\left(h_{i}\right)\right)\right| \\
& \leq d_{\mathrm{rk}}\left(\xi\left(g_{i}\right), \xi\left(h_{i}\right)\right) \leq \sum_{j=0}^{k} d_{\mathrm{rk}}\left(a_{j} g_{i}^{j}, a_{j} h_{i}^{j}\right) \leq \sum_{j=0}^{k} d_{\mathrm{rk}}\left(g_{i}^{j}, h_{i}^{j}\right) \\
& \leq\left(\sum_{j=0}^{k} j\right) d_{\mathrm{rk}}\left(g_{i}, h_{i}\right)=\left(\begin{array}{c}
k+1 \\
2
\end{array}\right) d_{\mathrm{rk}}\left(g_{i}, h_{i}\right),
\end{aligned}
$$

and the latter tends to zero along $\mathcal{U}$. Here we used the same trick as in estimate (3.1) above to bound $d_{\mathrm{rk}}\left(g_{i}^{j}, h_{i}^{j}\right)$ by $j d_{\mathrm{rk}}\left(g_{i}, h_{i}\right)(j=0, \ldots, k)$ in esti-

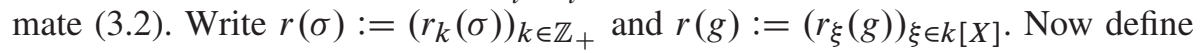


$q_{k}(\sigma)$ for $k \in \mathbb{Z}_{+}$via the equality

$$
\sum_{d \mid k} q_{d}(\sigma)=r_{k}(\sigma) \quad \text { for all } k \in \mathbb{Z}_{+} .
$$

Write $q(\sigma):=\left(q_{k}(\sigma)\right)_{k \in \mathbb{Z}_{+}}$. Applying Möbius inversion, we obtain

$$
q_{k}(\sigma)=\sum_{d \mid k} \mu(k / d) r_{d}(\sigma)
$$

Alternatively, one can think of $q_{k}(\sigma)$ as the $U$-limit of the normalized cardinality of the support of all $k$-cycles in $\sigma_{i}(i \in I)$, i.e.,

$$
q_{k}(\sigma)=\lim _{u} n_{k}\left(\sigma_{i}\right) / n_{i}=k \lim _{u} c_{k}\left(\sigma_{i}\right) / n_{i} .
$$

Similarly, for $\chi=i^{e} \in k[X]$ primary, define $q_{\chi}(g)$ via the equality

$$
r_{\xi}(g)=\sum_{\chi \text { primary }} \frac{\operatorname{deg}(\operatorname{gcd}\{\chi, \xi\})}{\operatorname{deg}(\chi)} q_{\chi}(g)
$$

for all polynomials $\xi \in k[X]$. Here $\operatorname{gcd} S$ is the greatest common divisor of the elements from $S$. Write $q(g):=\left(q_{\chi}(g)\right)_{\chi}$ primary. Alternatively, one can think of $q_{\chi}(g)$ as the $U$-limit of the normalized dimensions of the (not unique) subspaces $V_{\chi}\left(g_{i}\right)(i \in I)$, i.e.,

$$
q_{\chi}(g)=\lim _{u} n_{\chi}\left(g_{i}\right) / n_{i}=k_{\chi} \lim _{u} c_{\chi}\left(g_{i}\right) / n_{i},
$$

where $k_{\chi}=\operatorname{deg}(\chi)=e \operatorname{deg}(i)$. This is because, when $g$ acts as $F(\chi)$ and $\xi \in k[X]$, then $\operatorname{dim}(\operatorname{ker}(\xi(g)))=\operatorname{deg}(\operatorname{gcd}\{\chi, \xi\})$.

We claim that the conjugacy classes in $\mathrm{S} u$ resp. $\mathbf{M}_{\mathcal{U}}(q)$ are in one-to-one correspondence with all tuples $\left(q_{k}(\sigma)\right)_{k \in \mathbb{Z}_{+}}$resp. $\left(q_{\chi}(g)\right)_{\chi}$ primary, where the only condition on the sequences are that

$$
\sum_{k \in \mathbb{Z}_{+}} q_{k}(\sigma) \leq 1 \quad \text { resp. } \quad \sum_{\chi \text { primary }} q_{\chi}(g) \leq 1 .
$$

Here we let GL $u(q)$ act on $\mathbf{M}_{U}(q)$ by conjugation. The element $g$ lies in GLu(q) iff $q_{\chi}(g)=0$ for $\chi=X^{e}(e \geq 1)$. Indeed, one sees easily that $r(\sigma)$ resp. $r(g)$ is conjugacy invariant, and so is $q(\sigma)$ resp. $q(g)$ for $\sigma \in \mathrm{S}_{\mathcal{U}}$ and $g \in \mathbf{M}_{\mathcal{U}}(q)$.

To see the converse, let

$$
\begin{aligned}
\sigma & ={\overline{\left(\sigma_{i}\right)}}_{i \in I}, \tau={\overline{\left(\tau_{i}\right)}}_{i \in I} \in \mathrm{S}_{u}, \\
g & ={\overline{\left(g_{i}\right)_{i \in I}}}_{i}, h={\overline{\left(h_{i}\right)}}_{i \in I} \in \mathbf{M}_{u}(q)
\end{aligned}
$$

be elements such that $q(\sigma)=q(\tau)$ and $q(g)=q(h)$, respectively. 
Find a sequence $\left(N_{i}\right)_{i \in I}$ tending to infinity along $\mathcal{U}$ such that

$$
\sum_{k=1}^{N_{i}}\left|q_{k}(\sigma)-q_{k}\left(\sigma_{i}\right)\right|, \sum_{k=1}^{N_{i}}\left|q_{k}(\tau)-q_{k}\left(\tau_{i}\right)\right| \rightarrow u 0
$$

resp. such that

$$
\sum_{\substack{\chi \text { primary } \\ \operatorname{deg}(\chi) \leq N_{i}}}\left|q_{\chi}(g)-q_{\chi}\left(g_{i}\right)\right|, \sum_{\substack{\chi \text { primary } \\ \operatorname{deg}(\chi) \leq N_{i}}}\left|q_{\chi}(h)-q_{\chi}\left(h_{i}\right)\right| \rightarrow u 0 .
$$

Then, by the triangle inequality,

$$
\frac{1}{n_{i}} \sum_{k=1}^{N_{i}}\left|n_{k}\left(\sigma_{i}\right)-n_{k}\left(\tau_{i}\right)\right| \rightarrow u 0
$$

resp.

$$
\frac{1}{n_{i}} \sum_{\substack{\chi \text { primary } \\ \operatorname{deg}(\chi) \leq N_{i}}}\left|\operatorname{dim}\left(V_{\chi}\left(g_{i}\right)\right)-\operatorname{dim}\left(V_{\chi}\left(h_{i}\right)\right)\right| \rightarrow u 0 .
$$

Hence we can conjugate a big part of $\bigsqcup_{k=1}^{N_{i}} \Omega_{k}\left(\sigma_{i}\right)$ equivariantly to a big part of $\bigsqcup_{k=1}^{N_{i}} \Omega_{k}\left(\tau_{i}\right)$ resp. an almost full-dimensional part of

$$
\bigoplus_{\substack{\chi \text { primary } \\ \operatorname{deg}(\chi) \leq N_{i}}} V_{\chi}\left(g_{i}\right) \text { to } \bigoplus_{\substack{\chi \text { primary } \\ \operatorname{deg}(\chi) \leq N_{i}}} V_{\chi}\left(h_{i}\right)
$$

equivariantly (with no error in the limit; here again $V_{\chi}\left(g_{i}\right)$ resp. $V_{\chi}\left(h_{i}\right)$ are not unique). The remaining part of $\sigma_{i}$ and $\tau_{i}$ resp. $g_{i}$ and $h_{i}$ can be modified into one big cycle resp. one big Frobenius block with no change of $\sigma$ and $\tau$ resp. $g$ and $h$, since $N_{i} \rightarrow u \infty$. Then we conjugate this cycle of $\sigma_{i}$ resp. Frobenius block of $g_{i}$ onto the one of $\tau_{i}$ resp. $h_{i}$.

The case of $\mathrm{PM}_{U}(q)$. Let the group $k^{\times}=\mathbb{F}_{q}^{\times}$act on all (monic) polynomials $\xi \in k[X]$ by $\xi . z:=z^{-k_{\xi}} \xi(z X)$, where $k_{\xi}:=\operatorname{deg}(\xi)$. Extend this action to all tuples $q=\left(q_{\chi}\right)_{\chi}$ primary with $\sum_{\chi \text { primary }} q_{\chi} \leq 1$ via

$$
q . z=\left(q_{\chi . z}\right)_{\chi} \text { primary }
$$

and denote by $\bar{q}$ the orbit $\operatorname{orb}_{k^{\times}}(q)$ of $q$ under this action of $k^{\times}$.

Suppose that $\bar{G}=$ PGL $_{U}(q)$. We claim that the conjugacy classes of elements

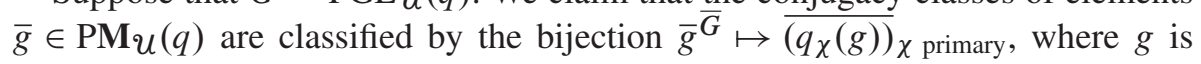
any lift of $\bar{g}$ in $\mathbf{M}_{u}(q)$ (here we exclude the tuple $q$ where $q_{X}=1$ and $q_{\chi}=0$ otherwise). 
Indeed, the map is well-defined since any other $h$ such that $\bar{h}=\bar{g} \in \operatorname{PM}_{u}(q)$ is of the form $z g$ for some $z \in k^{\times}$(as $k=\mathbb{F}_{q}$ is finite) so that

$$
\left(q_{\chi}(h)\right)_{\chi \text { primary }}=\left(q_{\chi}(z g)\right)_{\chi \text { primary }}=\left(q_{\chi . z}(g)\right)_{\chi \text { primary }}=q(g) . z .
$$

Also $q$ is constant on conjugacy classes of $\mathbf{M}_{\mathcal{U}}(q)$ (under the action of GLU $(q)$ ). Conversely, if $q_{\chi}(h)=q_{\chi . z}(g)=q_{\chi}(z g)$ for some fixed $z \in k^{\times}$and all $\chi \in k[X]$ primary, then, from the above, we derive that the elements $g$ and $z^{-1} h$ of $\mathbf{M}_{U}(q)$ are conjugate so that $\bar{g}$ and $\bar{h}$ are conjugate in $\mathrm{PM}_{u}(q)$. This proves the claim.

Remark 2. For $G$ of type $\operatorname{Sp}_{u}(q), \mathrm{GO}_{u}(q)$, or $\mathrm{GU}_{u}(q)$, the conjugacy classes of elements $g \in G$ for which $\sum_{\chi \text { primary }} q_{\chi}(g)=1$ are still characterized by the tuples $\left(q_{\chi}(g)\right)_{\chi}$ primary. The only necessary additional restriction on these tuples is that $q_{\chi}(g)=q_{\chi^{*}}(g)$ for all $\chi \in k[X]$ primary (see the beginning of Section 2 for the definition of $\left.\chi^{*}\right)$.

Indeed, assume $g={\overline{\left(g_{i}\right)}}_{i \in I}, h=\overline{(h}_{i}{ }_{i \in I} \in G, q(g)=q(h), q_{\chi}(g)=q_{\chi^{*}}(g)$ for all $\chi \in k[X]$ primary, and

$$
\sum_{\chi \text { primary }} q_{\chi}(g)=\sum_{\chi \text { primary }} q_{\chi}(h)=1 .
$$

Then $g$ is conjugate to $h$.

This holds since on all but constantly many Frobenius blocks $F(\chi)$ of $g_{i}$ resp. $h_{i}(i \in I)$, where $\chi$ is a self-dual primary polynomial, and on all but constantly many blocks $F(\chi) \oplus F\left(\chi^{*}\right) \cong F\left(\chi \chi^{*}\right)$, where $\chi$ is not self-dual and primary, according to Fact 1 , the form $f_{i}$ (and in characteristic $p=2$ the quadratic form $Q_{i}$ ) is uniquely determined up to linear equivalence so that we can map these blocks of $g_{i}$ to such blocks of $h_{i}$ and extend this partial map by Witt's lemma.

Conversely, if we have a tuple $\left(q_{\chi}\right)_{\chi}$ primary such that $\sum_{\chi}$ primary $q_{\chi}=1$ and $q_{\chi}=q_{\chi^{*}}$, we can deduce again from Fact 1 that there exists an element $g \in G$ such that $q_{\chi}(g)=q_{\chi}$ for all $\chi \in k[X]$ primary.

Recall that $\bar{G}=G / Z$, where $Z=\mathbf{Z}(G)$. For a tuple $q=\left(q_{\chi}\right)_{\chi}$ primary, let $\bar{q}$ denote its orbit $\operatorname{orb}_{Z}(q)$ under the action of $Z \leq k^{\times}$. Then, for the elements $\bar{g} \in \bar{G}$ (i.e., $\bar{G}$ is $\operatorname{PSp}_{u}(q)$, $\mathrm{PGO}_{u}(q)$, or $\left.\mathrm{PGU}_{u}(q)\right)$ such that $\sum_{\chi \text { primary }} q_{\chi}(g)=1$ for one lift $g \in G$ of $\bar{g}$, the same characterization as for PGL $\mathcal{U}(q)$ above holds by the same argument. Again we need to restrict the tuples $q=\left(q_{\chi}\right)_{\chi}$ primary so that $q_{\chi}=q_{\chi}$ for all $\chi$ primary.

However, we conjecture that the above characterization for $G$ of type $\operatorname{Sp}_{\mathcal{U}}(q)$, $\mathrm{GO}_{u}(q)$, or $\mathrm{GU}_{\mathcal{u}}(q)$ is false if

$$
\sum_{\chi \text { primary }} q_{\chi}(g)<1 \quad \text { for an element } g \in G .
$$


Remark 3. It is easy to see that $(\chi \cdot z)^{*}=\chi^{*} . z$ for $z \in Z \leq k^{\times}$. Indeed, since $z^{\sigma}=z^{-1}$ for $z \in Z$, we have

$$
\begin{aligned}
(\chi . z)^{*} & =\left(z^{-k} \chi(z X)\right)^{*}=\left(z^{k}\right)^{\sigma} a_{0}^{-\sigma} X^{k}\left(z^{-k}\right)^{\sigma} \chi^{\sigma}\left(z^{\sigma} X^{-1}\right) \\
& =a_{0}^{-\sigma} X^{k} \chi^{\sigma}\left((z X)^{-1}\right)=z^{-k} \chi^{*}(z X)=\chi^{*} . z,
\end{aligned}
$$

where $\chi=a_{0}+a_{1} X+\cdots+a_{k-1} X^{k-1}+X^{k}$ (recall that $\sigma \in \operatorname{Aut}(k)$ is defined in Section 2).

\section{Characterization of torsion elements in $\mathrm{S}_{u}, \mathrm{GL}_{u}(q)$, and PGLu(q)}

In this section, we characterize torsion elements in metric ultraproducts of the above type. At first, note that an invertible element in $\mathbf{M}_{u}(q)$, i.e., an element of GL $u(q)$, is algebraic over $k=\mathbb{F}_{q}$ if and only if it is torsion. Indeed, if $g$ is torsion, then $g^{o}-1=0$ for some integer $o \geq 1$. Conversely, if $g$ is algebraic and invertible, let $\chi \in k[X]$ be its minimal polynomial. Setting $o:=\left|(k[X] /(\chi))^{\times}\right|<\infty$, one sees that $g^{o}=1$ as $g$ is invertible.

Here comes the promised characterization of torsion elements.

Lemma 1. An element $\sigma \in \mathrm{S}_{\mathcal{U}}$ resp. $g \in \mathrm{GLu}(q)$ is torsion if and only if there is $N \in \mathbb{Z}_{+}$such that

$$
\sum_{k=1}^{N} q_{k}(\sigma)=1 \quad \text { resp. } \quad \sum_{\substack{\chi \text { primary } \\ \operatorname{deg}(\chi) \leq N}} q_{\chi}(g)=1 .
$$

An element $\bar{g} \in \mathrm{PGL} U(q)$ is torsion if and only if any lift $g \in \mathrm{GL} U(q)$ is torsion.

Proof. Write $1 \mathrm{~cm} S$ for the least common multiple of the elements of $S$. Indeed, if the above two conditions are fulfilled, then writing $o:=\operatorname{lcm}\{1, \ldots, N\}$ resp. $o:=\operatorname{lcm}\left\{\left|(k[X] /(\chi))^{\times}\right| \mid \chi\right.$ primary, $\left.\operatorname{deg}(\chi) \leq N\right\}$, we have $\ell_{\mathrm{H}}\left(\sigma_{i}^{o}\right) \rightarrow u 0$ resp. $\ell_{\mathrm{rk}}\left(g_{i}^{o}\right) \rightarrow u 0$, meaning that $\sigma^{o}=1$ resp. $g^{o}=1$.

Conversely, if we assume $\sigma^{o}=1$ resp. $g^{o}=1$, we get that $\ell_{\mathrm{H}}\left(\sigma_{i}^{o}\right) \rightarrow u 0$ resp. $\ell_{\mathrm{rk}}\left(g_{i}^{o}\right) \rightarrow u 0$, meaning that, asymptotically, the $d$-cycles in $\sigma_{i}$ for $d \mid o$ support the whole set resp. all Frobenius blocks $F(\chi)$ for $\chi \mid X^{o}-1$ primary support the whole vector space, so taking $N:=o$ above, we get the converse direction.

The last statement follows since the kernel of the surjective homomorphism $\mathrm{GL}_{\mathcal{U}}(q) \rightarrow$ PGL$U(q)$ equals $k^{\times}=\mathbb{F}_{q}^{\times}$, which is finite. Hence, if $g \in \mathrm{GL}_{\mathcal{U}}(q)$ represents $\bar{g} \in \operatorname{PGL} u(q)$ and the latter is of order $o<\infty$, then we have that $\operatorname{ord}(g) \mid o(q-1)<\infty$. 


\section{Faithful action of $\mathrm{S}_{u}$ and PGL $u(q)$ on the Loeb space and the associated continuous geometry}

In this section, we show that the groups $\mathrm{S}_{\mathcal{U}}$ and PGL $\mathcal{U}(q)$ faithfully act on natural associated objects. For this purpose, we need the so-called Loeb space

$$
L\left(n_{i}\right)_{i \in I}:=(\delta, \mu)
$$

resp. its vector space analog, the continuous geometry

$$
V\left(n_{i}\right)_{i \in I}:=(\mathcal{V}, \operatorname{dim})
$$

which are associated naturally to the metric ultraproduct $\mathrm{S}_{\mathcal{U}}$ resp. PGL $u(q)$.

Here $\&$ resp. $\mathcal{V}$ equals $\prod_{i \in I} \mathcal{P}\left(n_{i}\right)$ resp. $\prod_{i \in I} \operatorname{Sub}\left(k^{n_{i}}\right)$ modulo the equivalence relation $\left(S_{i}\right)_{i \in I} \sim\left(T_{i}\right)_{i \in I}$ resp. $\left(U_{i}\right)_{i \in I} \sim\left(V_{i}\right)_{i \in I}$ iff $\mu_{i}\left(S_{i} \triangle T_{i}\right) \rightarrow u 0$ resp. $\operatorname{dim}_{i}\left(U_{i}+V_{i}\right)-\operatorname{dim}_{i}\left(U_{i} \cap V_{i}\right) \rightarrow u 0$, where $\mu_{i}$ resp. $\operatorname{dim}_{i}$ is the normalized counting measure resp. dimension on $n_{i}$ resp. $k^{n_{i}}$ (and $A \triangle B$ denotes the symmetric difference of the sets $A$ and $B$ ). Then one defines $\mu$ resp. dim by

$$
\begin{gathered}
\mu(S)=\mu\left({\overline{\left(S_{i}\right)}}_{i \in I}\right):=\lim _{\mathcal{u}} \mu_{i}\left(S_{i}\right), \\
\operatorname{dim}(V)=\operatorname{dim}\left(\overline{\left.\left(V_{i}\right)_{i \in I}\right)}:=\lim _{u} \operatorname{dim}_{i}\left(V_{i}\right) .\right.
\end{gathered}
$$

It is easy to check that both are well-defined in this way. Also the operations $\cup, \cap$ resp.,$+ \cap$ are inherited to $\&$ resp. $\mathcal{V}$ in a natural way, e.g.,

$$
{\overline{\left(S_{i}\right)_{i}}}_{i \in I} \cap{\overline{\left(T_{i}\right)}}_{i \in I}:={\overline{\left(S_{i} \cap T_{i}\right)}}_{i \in I} .
$$

Write $S \subseteq T$ resp. $U \leq V$ iff $\mu(S \cap T)=\mu(S)$ resp. $\operatorname{dim}(U \cap V)=\operatorname{dim}(U)$. Call a permutation of $\delta$ resp. $\mathcal{V}$ an automorphism iff it preserves $\mu$ resp. dim and the relation $\subseteq$ resp. $\leq$.

Then one observes that $\mathrm{S} u$ resp. PGL $\mathcal{U}(q)$ is faithfully represented as group of automorphisms of $(\mathcal{S}, \mu)$ resp. $(\mathcal{V}, \operatorname{dim})$.

At first, consider the case $\bar{G}=\mathrm{S} u$. Indeed, assume for fixed $\sigma={\overline{\left(\sigma_{i}\right)_{i}}}_{i I} \in \mathrm{S} u$ that $S . \sigma=S$ for all $S \in \delta$. Then take $S={\overline{\left(S_{i}\right)}}_{i \in I}$, where $S_{i} \subseteq \underline{n_{i}}$ is taken in the following way. For each $k$-cycle $c \subseteq \underline{n_{i}}$ ( $k>1$; here seen as a set), we pick $s_{c} \in c$ and define $S_{i}$ by $S_{i} \cap c=\left\{s_{c}, s_{c} . \sigma_{i}^{2}, \ldots, s_{c} . \sigma_{i}^{2(\lfloor k / 2\rfloor-1)}\right\}$ and $S_{i} \cap \Omega_{1}\left(\sigma_{i}\right)=\emptyset$. Then $S_{i} \triangle S_{i} . \sigma_{i}=\emptyset$ and $\mu_{i}\left(S_{i}\right) \geq 1 / 3\left|\operatorname{supp}\left(\sigma_{i}\right)\right|$. This means that $S$ is fixed by $\sigma$ if and only if $\operatorname{supp}(\sigma):={\overline{\left(\operatorname{supp}\left(\sigma_{i}\right)\right)_{i \in I}}}_{i=\overline{(\varnothing)}}$ has measure zero. But this means $\sigma=$ id in the metric ultraproduct $\mathrm{S} u$.

Now we consider the case $\bar{G}=$ PGL $u(q)$. Here, similarly, assume for fixed $\left.g=\overline{(g}_{i}\right)_{i \in I} \in \mathrm{GL} u(q)$ that $V . g=V$ for all $V \in \mathcal{V}$. Then take $V={\overline{\left(V_{i}\right)}}_{i \in I}$ in 
the following way. The linear transformation $g_{i}$ is a direct sum of Frobenius blocks $F(\chi)$, where $\chi \in k[X]$ runs through all (monic) primary polynomials. For each such block $b \leq k_{i}^{n_{i}}$ of dimension $k_{b}>1$ (here seen as a subspace) of $g_{i}$ select a cyclic vector $v_{b}$. Then define $V_{i}$ by

$$
V_{i}=\bigoplus_{b, k_{b}>1}\left\langle v_{b}, v_{b} \cdot g_{i}^{2}, \ldots, v_{b} \cdot g_{i}^{2\left(\left\lfloor k_{b} / 2\right\rfloor-1\right)}\right\rangle .
$$

Then one observes that $V_{i} \cap V_{i} \cdot g_{i}=0$ so that

$$
\operatorname{dim}_{i}\left(V_{i}+V_{i} \cdot g_{i}\right)-\operatorname{dim}_{i}\left(V_{i} \cap V_{i} \cdot g_{i}\right)=2 \operatorname{dim}_{i}\left(V_{i}\right) .
$$

This shows that $q_{\chi}(g)=0$ for all $\chi \in k[X]$ primary of degree $k_{\chi}>1$. But one observes that, if $q_{(X-\lambda)}(g), q_{(X-\mu)}(g) \geq \varepsilon>0$ for $\lambda \neq \mu$ elements of $k$, we can use the following construction. Let

$$
e_{i 1}, \ldots, e_{i k_{i}} \in V_{X-\lambda}\left(g_{i}\right) \quad \text { and } \quad f_{i 1}, \ldots, f_{i k_{i}} \in V_{X-\mu}\left(g_{i}\right)
$$

such that $\lim _{u} k_{i} / n_{i}=\varepsilon$. Define $V_{i}:=\left\langle e_{i j}+f_{i j} \mid j=1, \ldots, k_{i}\right\rangle(i \in I)$. Assume $v \in V_{i} \cap V_{i} . g_{i}$. Then there exist numbers $\alpha_{1}, \ldots, \alpha_{k_{i}}, \beta_{1}, \ldots, \beta_{k_{i}} \in k$ such that

$$
v=\sum_{j=1}^{k_{i}} \alpha_{j}\left(e_{i j}+f_{i j}\right)=\sum_{j=1}^{k_{i}} \beta_{j}\left(\lambda e_{i j}+\mu f_{i j}\right) .
$$

This gives that

$$
\sum_{j=1}^{k_{i}}\left(\alpha_{j}-\beta_{j} \lambda\right) e_{i j}=\sum_{j=1}^{k_{i}}\left(\beta_{j} \mu-\alpha_{j}\right) f_{i j}
$$

so that by disjointness of $V_{X-\lambda}\left(g_{i}\right)$ and $V_{X-\mu}\left(g_{i}\right)$ both sides are zero and so, since the $e_{i j}, f_{i j}\left(j=1, \ldots, k_{i}\right)$ are linearly independent, we get that

$$
\alpha_{j}-\beta_{j} \lambda=\beta_{j} \mu-\alpha_{j}=0
$$

so that, since $\lambda \neq \mu$, we obtain $\alpha_{j}=\beta_{j}=0$. Hence $v=0$ and $V_{i} \cap V_{i} . g_{i}=0$. But $\lim u \operatorname{dim}\left(V_{i}\right) / n_{i} \geq \varepsilon$, yielding the same contradiction as above. Therefore, we must have $g=\lambda$ id (for $\lambda \in \mathbb{F}_{q}^{\times}$, as $k=\mathbb{F}_{q}$ is finite) in the metric ultraproduct $\operatorname{GL} u(q)$, i.e., $\operatorname{PGL} u(q)$ is faithfully represented.

Remark 4. The above statement about PGL $u(q)$ holds for any such metric ultraproduct of groups $\mathrm{PGL}_{n_{i}}\left(k_{i}\right)$ where the fields $k_{i}$ are not restricted with the same proof. Here the kernel of the action GLu $\rightarrow \operatorname{Aut}(\mathcal{V}, \operatorname{dim})$ is given by $\prod_{u} k_{i}^{\times}$(the algebraic ultraproduct of these groups). 
Remark 5. Hence, if the sequence of subsets $\left(S_{i}\right)_{i \in I}$ resp. subspaces $\left(V_{i}\right)_{i \in I}$ is almost stabilized by each element of a subgroup $H$ of $\bar{G}=\mathrm{S}_{u}$ resp. $\bar{G}=\operatorname{PGL} u(q)$ (or of $G=\mathrm{GL} u(q)$ ), we can restrict $H$ to $S:={\overline{\left(S_{i}\right)}}_{i \in I}$ resp. $V:={\overline{\left(V_{i}\right.}}_{i \in I}$.

Remark 6. For an element $\sigma={\overline{\left(\sigma_{i}\right)}}_{i \in I}$, set

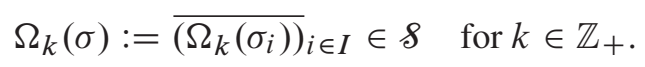

Similarly, for a semisimple element $g={\overline{\left(g_{i}\right)}}_{i \in I} \in \mathrm{GL} u(q)$ (see the next section for the definition of semisimple elements in such an ultraproduct), set

$$
V_{\chi}(g):={\overline{\left(V_{\chi}\left(g_{i}\right)\right)_{i \in I}}}_{\in \mathcal{V}} \text { for } \chi \in k[X] \text { irreducible. }
$$

Note that these definitions are independent of the chosen representatives (for the uniqueness of $V_{\chi}(g)$, we need that $g$ is semisimple since then $V_{\chi}\left(g_{i}\right)=\operatorname{ker}\left(\chi\left(g_{i}\right)\right)$ is unique for a suitable representative $\left(g_{i}\right)_{i \in I}$ of $g$ and $\chi \in k[X]$ irreducible).

Remark 7. Call a sequence of subsets $\left(B_{i}\right)_{i \in I} \subseteq k^{n_{i}}$ a basis of $V \in \mathcal{V}$ if there is a representative $\left(V_{i}\right)_{i \in I}$ of $V$ such that $B_{i}$ is a basis of $V_{i}(i \in I)$.

Remark 8. Call $V \in \mathcal{V}$ totally singular if it has a representative $\left(V_{i}\right)_{i \in I}$ such that each $V_{i}$ is totally singular (i.e., $\left.f_{i}\right|_{V_{i}}=0$ for $i \in I$ ).

\section{Centralizers in $\mathrm{S}_{u}, \mathrm{GL}_{u}(q), \mathrm{Sp}_{u}(q), \mathrm{GO}_{u}(q)$, and $\mathrm{GU}_{u}(q)$}

In this section, we provide tools (Lemmas 2 and 3) to compute centralizers of certain elements from the metric ultraproducts $\mathrm{S} u$ and GLu$u(q)$. We will use this in Section 7 to compute centralizers of elements in PGLu $(q)$. We write $\mathbf{C}(g)$ for the centralizer of the group element $g$.

Centralizers of elements in $G=\mathrm{S} u, \operatorname{GL} u(q)$. Note that, for $\sigma={\overline{\left(\sigma_{i}\right)}}_{i \in I} \in \mathrm{S} u$ resp. $g={\overline{\left(g_{i}\right)}}_{i \in I} \in \mathrm{GL} u(q)$, we have $\prod_{u} \mathbf{C}\left(\sigma_{i}\right) \leq \mathbf{C}(\sigma)$ resp. $\prod_{u} \mathbf{C}\left(g_{i}\right) \leq \mathbf{C}(g)$ (subsequently, by this notation we mean the metric ultraproduct of subgroups of the $H_{i}(i \in I)$ ). In the following lemma, we characterize when the above inclusion is actually an equality in the case of $\mathrm{S} u$.

Lemma 2. An element $\sigma \in \mathrm{S} u$ satisfies $\sum_{k \in \mathbb{Z}_{+}} q_{k}(\sigma)=1$ if and only if, for each choice of a representative $\left(\sigma_{i}\right)_{i \in I}$ of $\sigma$, the centralizer $\mathbf{C}(\sigma)$ equals $\prod_{u} \mathbf{C}\left(\sigma_{i}\right)$.

Before proving Lemma 2, we turn to $\operatorname{GL} u(q)$. An element $g \in \mathrm{GL}_{\mathcal{U}}(q)$ is called semisimple if it has a representative $\left(g_{i}\right)_{i \in I}$ such that each $g_{i} \in \mathrm{GL}_{n_{i}}(q)$ is semisimple, i.e., of order prime to $q$. 
Lemma 3. A semisimple element $g \in \mathrm{GL} u(q)$ satisfies $\sum_{\chi \text { primary }} q_{\chi}(g)=1$ if and only if for each choice of a representative $\left(g_{i}\right)_{i \in I}$ of $g$ where each $g_{i}$ is semisimple $(i \in I)$, the centralizer $\mathbf{C}(g)$ equals $\prod_{u} \mathbf{C}\left(g_{i}\right)$.

To prove Lemmas 2 and 3, we need the following auxiliary result.

Lemma 4. The following are true.

(i) Assume $\sigma \in \operatorname{Sym}(\underline{n})$ is of order $k$ and $S \subseteq \underline{n}$ has normalized counting measure $\mu(S)$. Then $S$ contains a $\sigma$-invariant subset $T$ of measure

$$
\mu(T) \geq 1-k(1-\mu(S))
$$

(ii) Assume $g \in \mathrm{GL}(V)$ for a $k$-vector space $V$ and that the minimal polynomial of $g$ over $k$ has degree $k$. Assume $U \leq V$. Then there exists a $g$-invariant subspace of $U$ of codimension at most $k \operatorname{codim}(U)$.

Proof. (i) Observe that the biggest $\sigma$-invariant subset of $S$ is equal to

$$
T=\bigcap_{i \in \mathbb{Z}} S \cdot \sigma^{i}
$$

But since $\sigma^{k}=\mathrm{id}$ by assumption, we see that actually $T=\bigcap_{i=0}^{k-1} S . \sigma^{i}$. Hence, since $\mu\left(S . \sigma^{i}\right)=\mu(S)$ for all $i \in \mathbb{Z}$, we have that $\mu(T) \geq 1-k(1-\mu(S))$.

(ii) Similarly to the above, the biggest $g$-invariant subspace contained in $U$ is $W=\bigcap_{i \in \mathbb{Z}} U . g^{i}$. Now $v \in \bigcap_{i=0}^{k-1} U . g^{i}$ means that $v, \ldots, v . g^{-(k-1)} \in U$. But then

$$
v \cdot g^{-k}=-\frac{1}{a_{0}}\left(a_{1} v \cdot g^{-(k-1)}+\cdots+a_{k-1} v \cdot g^{-1}+v\right) \in U,
$$

where $\chi=a_{0}+a_{1} X+\cdots+a_{k-1} X^{k-1}+X^{k}$ is the minimal polynomial of $g$. Note that $a_{0}=(-1)^{k} \operatorname{det}(g) \neq 0$. This shows that actually $W=\bigcap_{i=0}^{k-1} U . g^{i}$ so that $\operatorname{codim}(W) \leq k \operatorname{codim}(U)$.

Remark 9. The bounds in Lemma 4 are sharp. For example, take $\sigma$ of type $\left(k^{c_{k}}\right)$, and set $n=c_{k} k$. Take $S$ of size $n-s$ such that, for precisely $s \leq c_{k} k$-cycles of $\sigma, S$ contains $k-1$ elements of each of them and all elements of the remaining $k$-cycles. Then the set $T$ constructed in Lemma 4 has size $n-k s$. In (ii), we can use a similar construction.

Now we are able to prove Lemmas 2 and 3. 
Proof of Lemmas 2 and 3. At first, we prove Lemma 2. Assume that $\sigma={\overline{\left(\sigma_{i}\right)}}_{i \in I}$, $\tau={\overline{\left(\tau_{i}\right)}}_{i \in I} \in \mathrm{S} u$ commute, and assume that $\sum_{k=1}^{\infty} q_{k}(\sigma)=1$. Find a sequence $\left(N_{i}\right)_{i \in I}$ tending to infinity along $\mathcal{U}$ such that

$$
\lim _{u} \sum_{k=1}^{N_{i}} q_{k}\left(\sigma_{i}\right)=1 \quad \text { and }\left(\begin{array}{c}
N_{i}+1 \\
2
\end{array}\right) \ell_{\mathrm{H}}\left(\left[\sigma_{i}, \tau_{i}\right]\right) \rightarrow u 0 .
$$

Recall that $C_{k}\left(\sigma_{i}\right)$ denotes the set of $k$-cycles of the permutation $\sigma_{i}$. Call a $k$-cycle of $\sigma_{i}$ bad if it is not mapped $\sigma_{i}$-equivariantly to another $k$-cycle of $\sigma_{i}$ by $\tau_{i}$. Collect the set of bad $k$-cycles of $\sigma_{i}$ in $C_{k}^{\prime}\left(\sigma_{i}\right)$. For each bad $k$-cycle of $\sigma_{i}$, we get at least one non-fixed point of $\left[\sigma_{i}, \tau_{i}\right]$ so that $\left|C_{k}^{\prime}\left(\sigma_{i}\right)\right| / n_{i} \leq \ell_{\mathrm{H}}\left(\left[\sigma_{i}, \tau_{i}\right]\right)$ for all $k \in \mathbb{Z}_{+}$. Hence, if we change $\tau_{i}$ such that all bad $k$-cycles of $\sigma_{i}$ are mapped accurately for $k \leq N_{i}$ and all $k$-cycles for $k>N_{i}$ are mapped identically, we get a permutation $\tau_{i}^{\prime}$ such that

$$
\begin{aligned}
d_{\mathrm{H}}\left(\tau_{i}, \tau_{i}^{\prime}\right) & \leq \frac{1}{n_{i}} \sum_{k=1}^{N_{i}} k\left|C_{k}^{\prime}\left(\sigma_{i}\right)\right|+\sum_{k=N_{i}+1}^{\infty} q_{k}\left(\sigma_{i}\right) \\
& \leq\left(\sum_{k=1}^{N_{i}} k\right) \ell_{\mathrm{H}}\left(\left[\sigma_{i}, \tau_{i}\right]\right)+\sum_{k=N_{i}+1}^{\infty} q_{k}\left(\sigma_{i}\right) \\
& =\left(\begin{array}{c}
N_{i}+1 \\
2
\end{array}\right) \ell_{\mathrm{H}}\left(\left[\sigma_{i}, \tau_{i}\right]\right)+\sum_{k=N_{i}+1}^{\infty} q_{k}\left(\sigma_{i}\right) .
\end{aligned}
$$

By the assumption $\sum_{k=1}^{\infty} q_{k}(\sigma)=1$, the last term in the above estimate tends to

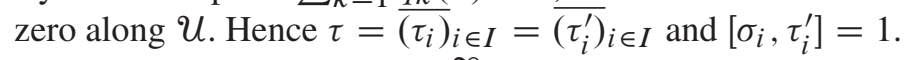

Conversely, assume that $\sum_{k=1}^{\infty} q_{k}(\sigma)<1$. Choose the sequence $\left(N_{i}\right)_{i \in I}$ such that $\lim _{u} \sum_{k=1}^{N_{i}} q_{k}\left(\sigma_{i}\right)=\sum_{k=1}^{\infty} q_{k}(\sigma)$ and $\lim _{u} N_{i} / n_{i}=0$.

For each $i \in I$, change $\sigma_{i}$ to $\sigma_{i}^{\prime}$ such that the $k$-cycles of $\sigma_{i}^{\prime}$ are the same as in $\sigma_{i}$ for $1 \leq k \leq N_{i}$ and the other $k$-cycles of $\sigma_{i}^{\prime}\left(k>N_{i}\right.$, if they exist) are grouped into one big $K_{i}$-cycle so that $d_{\mathrm{H}}\left(\sigma_{i}, \sigma_{i}^{\prime}\right)$ is minimal possible. It is easy to see that then still $d_{\mathrm{H}}\left(\sigma_{i}, \sigma_{i}^{\prime}\right) \rightarrow u 0$ as $N_{i} \rightarrow u \infty$. Now $\sigma_{i}^{\prime}$ eventually has precisely one $K_{i}$-cycle for $K_{i}>N_{i}$. Obtain $\sigma_{i}^{\prime \prime}$ by dividing this $K_{i}$-cycle (if it exists) into two $\left\lfloor K_{i} / 2\right\rfloor$-cycles and at most one fixed point so that $d_{\mathrm{H}}\left(\sigma_{i}^{\prime}, \sigma_{i}^{\prime \prime}\right) \leq 3 / n_{i}$ is minimal. Note that $K_{i} / n_{i}=1-\sum_{k=1}^{N_{i}} q_{k}\left(\sigma_{i}\right) \rightarrow u \varepsilon>0$ so that $\left\lfloor K_{i} / 2\right\rfloor>N_{i}$ along $\mathcal{U}$, as $\lim _{u} N_{i} / n_{i} \rightarrow u 0$ by assumption.

Now consider the restriction of the centralizers $\mathbf{C}\left(\sigma_{i}^{\prime}\right)$ and $\mathbf{C}\left(\sigma_{i}^{\prime \prime}\right)$ to the support of the unique $K_{i}$-cycle of $\sigma_{i}^{\prime}$ (which certainly both fix setwise by the previous inequality). The first group is isomorphic to the cyclic group $\mathrm{C}_{K_{i}}$, whereas the 
second is isomorphic to $\mathrm{C}_{\left\lfloor K_{i} / 2\right\rfloor}\left\langle\mathrm{C}_{2}\right.$. Taking the metric ultraproducts of these groups restricted to this support (in the sense of Remark 5), we get an abelian group in the first case, and a non-abelian group in the second case. Hence, in at least one case, $\prod_{u} \mathbf{C}\left(\sigma_{i}^{\prime}\right) \neq \mathbf{C}(\sigma)$ or $\prod_{u} \mathbf{C}\left(\sigma_{i}^{\prime \prime}\right) \neq \mathbf{C}(\sigma)$.

Now we prove Lemma 3. Assume that $g={\overline{\left(g_{i}\right)}}_{i \in I}, h={\overline{\left(h_{i}\right)}}_{i \in I} \in \operatorname{GL} u(q)$ commute, i.e., $[g, h]=\mathrm{id}$, that $g$ and each $g_{i}(i \in I)$ is semisimple, and assume that

$$
\sum_{\chi \text { irreducible }}^{\infty} q_{\chi}(g)=1
$$

Recall that semisimplicity means that, for each Frobenius block $F(\chi)$ in the generalized Jordan normal form of $g_{i}, \chi=i^{1}$ is irreducible. Choose the sequence $\left(N_{i}\right)_{i \in I}$ such that

$$
\lim _{u} \sum_{\substack{\chi \text { irreducible } \\
\operatorname{deg}(\chi) \leq N_{i}}} q_{\chi}\left(g_{i}\right)=1 \text { and }\left(\sum_{\begin{array}{c}
\chi \text { irreducible } \\
\operatorname{deg}(\chi) \leq N_{i}
\end{array}} \operatorname{deg}(\chi)\right) \ell_{\mathrm{rk}}\left(\left[g_{i}, h_{i}\right]\right) \rightarrow u 0 .
$$

Define $U_{i}:=\operatorname{ker}\left(\left[g_{i}, h_{i}\right]-\mathrm{id}\right)$. Fix an irreducible polynomial $\chi \in k[X]$ and apply Lemma 4 (ii) inside $V:=V_{\chi}\left(g_{i}\right)$ to the subspace $U:=U_{i} \cap V_{\chi}\left(g_{i}\right)$ to get a $g_{i}$-invariant subspace $W=W_{i \chi} \leq U$ such that $\operatorname{codim}_{V}\left(W_{i \chi}\right) \leq k_{\chi} \operatorname{codim}\left(U_{i}\right)$, where $k_{\chi}=\operatorname{deg}(\chi)$. Note here that $V_{\chi}\left(g_{i}\right)=\operatorname{ker}\left(\chi\left(g_{i}\right)\right)$ is unique since $g_{i}$ is semisimple. This large-dimensional subspace $W_{i \chi}$ is mapped accurately by $h_{i}$, as $g_{i}$ commutes with $h_{i}$ on it. Define $h_{i}^{\prime}$ to be equal to $h_{i}$ on each $W_{i \chi}$, and complete it on each $V_{i \chi}$ to a map commuting with $g_{i}$ for $\operatorname{deg}(\chi) \leq N_{i}$ (here we use semisimplicity of $\left.g_{i}\right)$. On $V_{\chi}\left(g_{i}\right)$ with $\operatorname{deg}(\chi)>N_{i}$, set $h_{i}^{\prime}$ to be the identity. As in the proof for $\mathrm{S} u$ above, it follows that $d_{\mathrm{rk}}\left(h_{i}, h_{i}^{\prime}\right) \rightarrow u 0$ and $\left[g_{i}, h_{i}^{\prime}\right]=1$.

Conversely, assume that $\sum_{\chi}^{\infty}$ irreducible $q_{\chi}(g)<1$. Choose the sequence $\left(N_{i}\right)_{i \in I}$ such that

$$
\lim _{\mathcal{u}} \sum_{\substack{\chi \text { irreducible } \\ \operatorname{deg}(\chi) \leq N_{i}}}^{N_{i}} q_{\chi}\left(g_{i}\right)=\sum_{\chi \text { irreducible }}^{\infty} q_{\chi}(g) \text { and } \lim _{\mathcal{u}} N_{i} / n_{i}=0 .
$$

For each $i \in I$, change $g_{i}$ into $g_{i}^{\prime}$ such that all Frobenius blocks $F(\chi)$ for $\chi$ irreducible of degree at most $N_{i}$ are left unchanged and all bigger Frobenius blocks (if there is any such block) are grouped into one big Frobenius block $F(\varphi)$ of size $K_{i}$ (for $\varphi$ irreducible). Define $g_{i}^{\prime \prime}$ in the same way, but split the Frobenius block $F(\varphi)$ (if it exists) into two or three blocks, two of which are $F(\phi)$ for $\phi$ irreducible of degree $\left\lfloor K_{i} / 2\right\rfloor$ and, if $K_{i}$ is odd, one block of size one, which is the identity. Then, as above, the centralizer of $g_{i}^{\prime}$ restricted to the large Frobenius block $F(\varphi)$ of it, 
equals $\mathbf{C}\left(g_{i}^{\prime}\right) \cong(k[X] /(\varphi))^{\times}$, whereas the centralizer $\mathbf{C}\left(g_{i}^{\prime \prime}\right)$ restricted to the same subspace is non-abelian (again in the sense of Remark 5). Also one sees that their metric ultraproducts are non-isomorphic, similarly to the case of permutations. The proof is complete.

Remark 10. If $G$ is one of $\operatorname{Sp}_{u}(q), \mathrm{GO}_{u}(q)$, or $\mathrm{GU}_{u}(q)$ and a semisimple $g \in G$ is represented by $\left(g_{i}\right)_{i \in I}$ and $\sum_{\chi}$ irreducible $q_{\chi}(g)=1$, one can adapt the above argument for $\mathrm{GL} u(q)$ to see that still $\mathbf{C}(g)=\prod_{u} \mathbf{C}\left(g_{i}\right)$ when all $g_{i}$ are semisimple.

Indeed, from Fact 3 , it follows that, in the space $W_{i \chi}+W_{i \chi^{*}}$ (where $W_{i \chi}, W_{i \chi^{*}}$ are constructed as above), we can still find a big, i.e., almost full-dimensional, $g_{i}$ invariant non-singular subspace $W_{i \chi, \chi^{*}}^{\prime}$. This is because each block $F(\chi)$ must be paired with a block $F\left(\chi^{*}\right)$ (which can possibly be the same as the first one) to get an indecomposable. Now $W_{i \chi}$ contains almost all blocks $F(\chi)$ of $g_{i}$, and $W_{i \chi^{*}}$ contains almost all blocks $F\left(\chi^{*}\right)$ of $g_{i}$, so many of them must be paired together.

By Fact 2, we can then complete the partial map

$$
\left.h_{i}\right|_{W_{i \chi, \chi^{*}}^{\prime}}: W_{i \chi, \chi^{*}}^{\prime} \rightarrow W_{i \chi, \chi^{*}}^{\prime} \cdot h_{i}
$$

to a map $h_{i}^{\prime} \in \mathrm{GL}\left(V_{\chi}\left(g_{i}\right)+V_{\chi^{*}}\left(g_{i}\right)\right)$ stabilizing $f_{i}$ (and $Q_{i}$ in the orthogonal case for $p=2$ ). So we have found an element $h_{i}^{\prime} \in H_{i}$ close to $h_{i}$ which commutes with $g_{i}$. This completes the proof.

As a consequence of Lemma 1 together with Lemmas 2 and 3, and Remark 10, we get the following corollary.

Corollary 1. If an element $\sigma \in \mathrm{S} u$ resp. a semisimple element

$$
g \in \operatorname{GL} u(q), \mathrm{Sp}_{u}(q), \mathrm{GO}_{u}(q) \text {, or } \mathrm{GU}_{u}(q)
$$

is torsion, then $\mathbf{C}(\sigma)$ resp. $\mathbf{C}(g)$ is equal to $\prod_{u} \mathbf{C}\left(\sigma_{i}\right)$ resp. $\prod_{u} \mathbf{C}\left(g_{i}\right)$ for each representative $\left(\sigma_{i}\right)_{i \in I}$ resp. $\left(g_{i}\right)_{i \in I}$ of $\sigma$ resp. $g$, where we require all $g_{i}(i \in I)$ to be semisimple.

\section{Centralizers in $\mathrm{PGL}_{u}(q), \mathrm{PSp}_{u}(q), \mathrm{PGO}_{u}(q)$, and $\mathrm{PGU}_{u}(q)$}

Now we can deduce the structure of centralizers of semisimple elements from PGL $u(q)$. By this we mean elements that lift to semisimple elements in GLu $(q)$. Let $g={\overline{\left(g_{i}\right)}}_{i \in I} \in \mathrm{GL} u(q)$ be a semisimple element which maps to

$$
\bar{g} \in \operatorname{PGL} u(q)=\operatorname{GL} u(q) / k^{\times} .
$$

All $g_{i}$ are assumed to be semisimple $(i \in I)$. 
Assume that $h=\overline{(h i)}_{i \in I} \in \mathrm{GL} u(q)$ is such that $[g, h]=\mu$ id for $\mu \in k^{\times}$. Then $g^{h}=\mu g$ so that $q(g)=q\left(g^{h}\right)=q(\mu g)=q(g)$. $\mu$, i.e., $\mu \in \operatorname{stab}_{k^{\times}}(q(g))$. Let $v \in \operatorname{stab}_{k^{\times}}(q(g)) \leq k^{\times}$be a generator of this cyclic group.

It is now easy to see that the conformal centralizer

$$
\mathbf{C}_{\text {conf }}(g):=\left\{h \in \operatorname{GL} u(q) \mid \text { there is } \mu \in k^{\times} \text {such that } g^{h}=\mu g\right\}
$$

is an extension $\mathbf{C}(g)$. $\operatorname{stab}_{k^{\times}}(q(g))=\mathbf{C}(g) .\langle v\rangle$ of $\mathbf{C}(g)$ by $\operatorname{stab}_{k^{\times}}(q(g))$. Hence $\mathbf{C}(\bar{g})=(\mathbf{C}(g) \cdot\langle v\rangle) / k^{\times}$.

Remark 11. The analog statement of Lemma 3 is false in PGL $u(q)$. Indeed, take a semisimple element $\bar{g} \in \mathrm{PGL} u(q)$ such that, for a lift $g \in \mathrm{GL}_{U}(q)$, the group $\operatorname{stab}_{k^{\times}}(q(g))$ is non-trivial. Choose a representative $\left(g_{i}\right)_{i \in I}$ of $g \in \mathrm{GL} u(q)$ such that $q_{\chi}\left(g_{i}\right) \neq q_{\xi}\left(g_{i}\right)$ for all $\chi, \xi \in k[X]$ distinct irreducible and $g_{i}$ is semisimple $(i \in I)$. Then $\mathbf{C}\left(g_{i}\right)$ stabilizes each subspace $V_{\chi}\left(g_{i}\right)=\operatorname{ker}\left(\chi\left(g_{i}\right)\right) \leq k^{n_{i}}$. But this means that, if $h \in C:=\prod_{u} \mathbf{C}_{\text {conf }}\left(g_{i}\right)$, we have that $g^{h}=g$ so that $C / k^{\times}$ is properly contained in $\mathbf{C}(\bar{g})$ (namely, $\mathbf{C}(\bar{g}) /\left(C / k^{\times}\right) \cong \operatorname{stab}_{k^{\times}}(q(g))$, which is non-trivial).

Remark 12. For the groups $\operatorname{PSp}_{u}(q)$, $\operatorname{PGO}_{u}(q)$, and PGU $u(q)$, the same structure for $\mathbf{C}(\bar{g})$ holds true, where $\mathrm{Sp}_{u}(q), \mathrm{GO}_{u}(q)$ resp. $\mathrm{GU} u(q)$ play the role of $\mathrm{GL}_{u}(q)$. The possible scalars $\mu \in k^{\times}$(from $Z$ ) are restricted to $\mu \in\{ \pm 1\}$ in the bilinear case, and to $\mu^{q+1}=1$ in the unitary case.

\section{Double centralizers of torsion elements}

In this section, we compute the double centralizers of semisimple torsion elements of the groups $\bar{G}$ of type $\mathrm{S} u, \mathrm{PGL}_{u}(q), \mathrm{PSp}_{u}(q), \mathrm{PGO}_{u}(q)$, and $\mathrm{PGU}_{u}(q)$. Note that, for $g \in G$ a group element, $\mathbf{C}(\mathbf{C}(g))=\mathbf{Z}(\mathbf{C}(g))$ since $g \in \mathbf{C}(g)$ so that $\mathbf{C}(\mathbf{C}(g)) \leq \mathbf{C}(g)$. Set $\mathbf{C}^{2}(g):=\mathbf{C}(\mathbf{C}(g))$ and $\mathbf{C}_{\text {conf }}^{2}(g):=\mathbf{C}_{\text {conf }}\left(\mathbf{C}_{\text {conf }}(g)\right)$ to be the double centralizer resp. double conformal centralizer of $g$. Here

$$
\mathbf{C}_{\text {conf }}(g):=\{h \in G \mid[g, h] \in \mathbf{Z}(G)\} .
$$

\subsection{The case $\mathrm{S}_{\boldsymbol{u}}$}

Let $\sigma={\overline{\left(\sigma_{i}\right)}}_{i \in I} \in \mathrm{S} u=\bar{G}$ be torsion of order $o$. Then we have $\sum_{k \mid o} q_{k}(\sigma)=1$ by Lemma 1. By Corollary 1 , we have that $\mathbf{C}(\sigma)=\prod_{u} \mathbf{C}\left(\sigma_{i}\right)$. But $\mathbf{C}\left(\sigma_{i}\right)$ has a subgroup

$$
\prod_{k \mid o} \mathrm{C}_{k} \prec \operatorname{Sym}\left(c_{k}\left(\sigma_{i}\right)\right)
$$

which is dense in it along $U$ so that $C:=\mathbf{C}(\sigma)=\prod_{u} \prod_{k \mid o} \mathrm{C}_{k} \prec \operatorname{Sym}\left(c_{k}\left(\sigma_{i}\right)\right)$. 
At first, for simplicity, assume that $\sigma_{i}$ is isotypic of type $\left(k^{c_{i k}}\right)$ (so $n_{i}=c_{i k} k$ ). Assume that $\tau={\overline{\left(\tau_{i}\right)}}_{i \in I} \in \mathbf{Z}(C)$ and $\tau_{i}=\left(a_{i j}\right) \cdot \varphi_{i} \in \mathrm{C}_{k}\left\langle\operatorname{Sym}\left(c_{i k}\right)\right.$. Assume that $\lim u\left|\operatorname{supp}\left(\varphi_{i}\right)\right| / c_{i k}=\varepsilon>0$. Then we can conjugate $\varphi_{i}$ by

$$
\phi_{i} \in \operatorname{Sym}\left(c_{i k}\right) \leq \mathrm{C}_{k} \prec \operatorname{Sym}\left(c_{i k}\right)=\mathbf{C}\left(\sigma_{i}\right)
$$

such that

$$
\lim _{\mathcal{U}} d_{\mathrm{H}}\left(\varphi_{i} \phi_{i}, \phi_{i} \varphi_{i}\right) \geq \varepsilon>0
$$

But this leads to the contradiction

$$
\lim _{u} d_{\mathrm{H}}\left(\tau_{i} \phi_{i}, \phi_{i} \tau_{i}\right) \geq \varepsilon>0
$$

Hence we may assume that $\varphi_{i}=\mathrm{id}$, applying a small change to $\tau_{i}$ along $\mathcal{U}$ if necessary $(i \in I)$. Now assume that $\lim u\left|\left\{j \mid a_{i j}=c\right\}\right| / c_{i k}=\varepsilon \in(0,1)$. Then we find permutations $\phi_{i} \in \operatorname{Sym}\left(c_{i k}\right) \leq \mathrm{C}_{k} 2 \operatorname{Sym}\left(c_{i k}\right)=\mathbf{C}\left(\sigma_{i}\right)$ such that

$$
d_{\mathrm{H}}\left(\tau_{i}, \tau_{i}^{\phi_{i}}\right)=\left|\left\{j \mid a_{i j} \neq a_{i j . \phi_{i}}\right\}\right| / c_{i k} \geq \min \{\varepsilon, 1-\varepsilon\}>0 .
$$

Hence we can assume that all $a_{i j}$ are equal. This shows that, in this case, $\mathbf{Z}(\mathbf{C}(\sigma))$ is the metric ultraproduct $\prod u \mathrm{C}_{k} \cong \mathrm{C}_{k}$, where $\mathrm{C}_{k}$ in the $i$ th component is generated by the element $\sigma_{i}$ itself $(i \in I)$.

In the general case, we obtain that

$$
\mathbf{Z}(\mathbf{C}(\sigma))=\prod_{k \mid o, q_{k}(\sigma)>0} \prod_{\mathcal{U}} \mathrm{C}_{k} \cong \prod_{k \mid o, q_{k}(\sigma)>0} \mathrm{C}_{k} .
$$

This holds because $\sigma \in \mathbf{C}(\sigma)$ so that, when $\tau \in \mathbf{Z}(\mathbf{C}(\sigma))$, it must commute with $\sigma$. But this implies that $\lim u\left|\Omega_{k}\left(\sigma_{i}\right) \triangle \Omega_{k}\left(\sigma_{i}\right) \cdot \tau_{i}\right|=0$ so that $\tau$ must stabilize the isotypic components of $\sigma$ (in the sense of Remark 5), and we can apply the above argument.

\subsection{The case PGLu(q), PSp $u(q), \mathrm{PGO}_{u}(q)$, and PGU $u(q)$}

Recall from Section 2 that $k=\mathbb{F}_{q}$ when $G$ is $\operatorname{GL}_{\mathcal{u}}(q), \operatorname{Sp}_{u}(q)$, or $\operatorname{GO}_{\mathcal{u}}(q)$, and $k=\mathbb{F}_{q^{2}}$ when $G=\operatorname{GU}_{U}(q)$. Set $d=1$ in the first three cases and $d=2$ when $G$ is unitary over $\mathbb{F}_{q^{2}}$.

Recall that $Z=k^{\times}$when $G=\operatorname{GL} u(q), Z=\{ \pm 1\} \subseteq k^{\times}$when $G=\operatorname{Sp}_{u}(q)$ or $G=\mathrm{GO}_{\mathcal{u}}(q)$, and $Z=\left\{z \in k^{\times} \mid z^{q+1}=1\right\} \subseteq k^{\times}=\mathbb{F}_{q^{2}}^{\times}$when $G=\operatorname{GU}_{u}(q)$. Also recall that, if $G$ is not of shape $\operatorname{GL} u(q)$, we have $z^{\sigma} \stackrel{q^{2}}{=} z^{-1}$ for $z \in Z$, where $\sigma: k \rightarrow k$ is the identity in the bilinear case, and the $q$-Frobenius endomorphism $x \mapsto x^{q}$ when $G=\operatorname{GU} u(q)$. Let $g={\overline{\left(g_{i}\right)}}_{i \in I} \in G \leq \operatorname{GL} u(k)$ be semisimple, 
with $g_{i}(i \in I)$ semisimple such that $\bar{g} \in \bar{G} \leq \mathrm{PGL} u(k)=\mathrm{GL} u(k) / k^{\times}$is torsion of order dividing $o$, i.e., there is $\mu \in k^{\times}$such that $g^{o}=\mu$ id. This implies $\mu \in Z$. Then

$$
\sum_{\substack{\chi \text { irreducible } \\ X^{o} \equiv \mu(\chi)}} q_{\chi}(g)=1
$$

by Lemma 1. Set

$$
P:=\left\{\chi \in k[X] \mid \chi \text { (monic) irreducible, } \chi \mid X^{o}-\mu\right\},
$$

$T:=\operatorname{stab}_{Z}(q(g)), K_{\chi}:=k[X] /(\chi)$ for $\chi \in k[X]$ irreducible (as in Fact 3), and $c_{i \chi}:=c_{\chi}\left(g_{i}\right)(i \in I)$. Hence, similarly to the above, we have

$$
\mathbf{C}(g)=\prod_{\substack { \chi \\
\begin{subarray}{c}{\chi \text { irreducible } \\
X^{o} \equiv \mu(\chi) \\
q_{\chi}(g)>0{ \chi \\
\begin{subarray} { c } { \chi \text { irreducible } \\
X ^ { o } \equiv \mu ( \chi ) \\
q _ { \chi } ( g ) > 0 } }\end{subarray}} \mathbf{M}_{c_{i \chi}}\left(K_{\chi}\right),
$$

the centralizer being computed in $\mathbf{M}_{u}(k)$. Now, by Section 7, we "know" the structure of $\mathbf{C}_{\text {conf }}(g) \leq G$. For $\chi \in k[X]$ irreducible, consider the $g$-invariant subspace $V:=V_{\bar{\chi}}(g):=\bigoplus_{\xi \in \bar{\chi}} V_{\xi}(g) \in \mathcal{V}$, where $\bar{\chi}:=\operatorname{orb}_{T}(\chi)$ is the orbit of $\chi$ un$\operatorname{der} T$ (see Remark 6 for the definition of $V_{\xi}(g) \in \mathcal{V}$ ). Set $l_{\chi}:=|\bar{\chi}|, m_{\chi}:=|T| / l_{\chi}$. Note that $m_{\chi}=\left|\operatorname{stab}_{T}(\chi)\right|$, and so

$$
m_{\chi}=\max \left\{m \text { divides }|T| \mid \text { there exists } \chi^{\prime} \text { such that } \chi=\chi^{\prime}\left(X^{m}\right)\right\} \text {. }
$$

The restriction of the action of $\mathbf{C}_{\text {conf }}(g) / Z$ to $V_{\bar{\chi}}(g)$ is given by

$$
\left(\left(\prod_{U} \prod_{\xi \in \bar{\chi}} \mathbf{C}\left(\left.g\right|_{V_{\xi}(g)}\right)\right) \cdot T\right) / Z .
$$

We will explain this below. In most cases, the above extension is even split.

Definition of the action of $T$. Let $t \in Z$ be a generator of the above cyclic group $T$. Then the map $\varphi_{t}$, which is constructed in the following, generates the above extension.

At first, note that we can see each space $V_{\xi}\left(g_{i}\right)$ as a vector space over the field $K_{\xi}=k[X] /(\xi)\left(\xi \in k[X]\right.$ irreducible) as $g$ acts on it as multiplication by $\lambda_{\xi}=\bar{X}$ acts on $K_{\xi}$ (cf. Fact 3). With this convention, find $K_{\xi}$-bases $\left(B_{\xi, i}\right)_{i \in I}$ of each $V_{\xi}(g)(\xi \in \bar{\chi}$ for all representatives $\chi$ of orbits of the action of $T$ on the irreducible polynomials; see Remark 7) and compatible bijections $\alpha_{\xi_{1}, \xi_{2}, i}: B_{\xi_{1}, i} \rightarrow B_{\xi_{2}, i}$ (for $i \in I$, i.e., $\alpha_{\xi_{2}, \xi_{3}, i} \circ \alpha_{\xi_{1}, \xi_{2}, i}=\alpha_{\xi_{1}, \xi_{3}, i}$ for all $\xi_{1}, \xi_{2}, \xi_{3} \in \bar{\chi}$, all $\chi$, and all $i \in I$ ). 
If $G$ comes from groups preserving a form, we still find bijections $\bullet *: B_{\xi, i} \rightarrow B_{\xi^{*}, i}$ such that $b^{* *}=b$, the pairing $f_{i}$ restricted to

$$
K_{\xi} b \times K_{\xi} b^{*}=k[g] b \times k[g] b^{*} \rightarrow k
$$

is non-singular, the pairing $f_{i}$ restricted to $K_{\xi} b \times K_{\xi^{\prime}} b^{\prime}$ is zero for all $b \in B_{\xi, i}$, $b^{\prime} \in B_{\xi^{\prime}, i}, b^{\prime} \neq b^{*}$, and such that $\bullet *$ commutes with the maps $\alpha_{\xi_{1}, \xi_{2}, i}(i \in I)$. Such bases exist by an application of Fact 3 . The last condition can be fulfilled since $(\xi . t)^{*}=\xi^{*} . t$ for all $\xi \in k[X]$ by Remark 3 .

Now we define maps $\varphi_{t, i}$ on $k^{n_{i}}$ by defining their restrictions

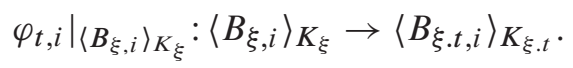

We do this by defining a $k$-linear map $\varphi_{\xi, t}: K_{\xi} \rightarrow K_{\xi . t}$ (which is not necessarily a homomorphism) and extending it to a map $\varphi_{t, i}$ by linearity,

$$
\varphi_{t, i}\left(\sum_{b \in B_{\xi, i}} \lambda_{b} b\right)=\sum_{b \in B_{\xi, i}} \varphi_{\xi, t}\left(\lambda_{b}\right) \alpha_{\xi, \xi . t, i}(b) .
$$

Let $\phi_{\xi, t}: K_{\xi} \rightarrow K_{\xi . t}$ denote the $k$-homomorphism such that $\lambda_{\xi} \mapsto t \lambda_{\xi . t}$. Here is our definition of $\varphi_{\xi, t}$.

If not $p>2, \varepsilon=-1, \chi=\chi^{*}, t=-1$, and $\chi . t=\chi$, set $\varphi_{\xi, t}:=\phi_{\xi, t}$. In this case, $\varphi_{t}^{|T|}=\mathrm{id}$, and the above extension is split.

In the opposite case,

$$
\xi=\xi^{*}=\xi . t=\xi .(-1) \quad \text { for all } \xi \in \bar{\chi} .
$$

Let $\eta \in K_{\xi}$ be a generator of the cyclic group $K_{\xi}^{\times}$and set $\zeta:=\eta^{\left(q^{k \chi / 2}-1\right) / 2}$, where $k_{\chi}=\operatorname{deg}(\chi)=\operatorname{deg}(\xi)$, so that $\xi^{q^{k} / 2}+1=-1$. Then set $\varphi_{\xi, t}: K_{\xi} \rightarrow K_{\xi . t}$ to be the map $x \mapsto \zeta \phi_{\xi, t}(x)$. In this case, $\varphi_{\xi, t}^{2}(x)=-x$, so the above extension needs not to be split.

Doing this for all representatives $\chi$ of orbits of the action of $T$ on the irreducible polynomials $\chi \in k[X]$ with $\chi \mid X^{o}-\mu$, this defines, up to a small error in the rank metric, a map $\varphi_{t, i}: k^{n_{i}} \rightarrow k^{n_{i}}$, so set $\varphi_{t}$ to be ${\overline{\left(\varphi_{t, i}\right)}}_{i \in I}$.

The map $g t$-commutes with $\varphi_{t}$. Recall from Fact 3 that $g_{i}(i \in I)$ acts on $K_{\xi}$ as multiplication by $\lambda_{\xi}$ (and so on $K_{\xi . t}$ as multiplication by $\lambda_{\xi . t}$ ). Hence, by the above definition of $\varphi_{t}$, we need to verify that

$$
\varphi_{\xi, t}\left(\lambda_{\xi} x\right)=t \lambda_{\xi . t} \varphi_{\xi, t}(x)
$$

for all $x \in K_{\xi}, \xi \in \bar{\chi}$. 
In the first of the above cases, this is true since then $\varphi_{\xi, t}=\phi_{\xi, t}$ is a field isomorphism (and hence multiplicative) and $\phi_{\xi, t}\left(\lambda_{\xi}\right)=t \lambda_{\xi . t}$ by definition. In the second case, the left-hand side of equation (8.1) evaluates to

$$
\varphi_{\xi, t}\left(\lambda_{\xi} x\right)=\zeta \phi_{\xi, t}\left(\lambda_{\xi} x\right)=\zeta \phi_{\xi, t}\left(\lambda_{\xi}\right) \phi_{\xi, t}(x)=\zeta t \lambda_{\xi . t} \phi_{\xi, t}(x),
$$

whereas the right-hand side is $t \lambda_{\xi . t} \varphi_{\xi, t}(x)=t \lambda_{\xi . t} \zeta \phi_{\xi, t}(x)$, so they are equal.

The action of $\varphi_{t}$ preserves the forms $f_{i}$ (and $Q_{i}$ in the orthogonal case when $p=2, i \in I)$. Assume $G$ is not $\mathrm{GL}_{\mathcal{U}}(q)$. Then one sees that $\varphi_{t}$ preserves the forms $f_{i}(i \in I)$. We verify this in the different cases of Fact 3 . Let $b \in B_{\xi, i}$ (for $\xi \in \bar{\chi}$ ), and consider the restriction of $f_{i}$ to $U \times U^{*}:=K_{\xi} b \times K_{\xi *} b^{*}$. Recall from Fact 3 that, in this situation, $\tau: K_{\xi^{*}} \rightarrow K_{\xi}$ is the homomorphism such that $\tau: \lambda_{\xi^{*}} \mapsto \lambda_{\xi}^{-1}$ and $\left.\tau\right|_{h}=\sigma$. Let $\tau^{\prime}: K_{\xi^{*} . t} \rightarrow K_{\xi . t}$ be the corresponding homomorphism such that $\lambda_{\xi^{*} . t} \mapsto \lambda_{\xi . t}^{-1}$ and $\left.\tau^{\prime}\right|_{k}=\sigma$.

In case (i) (a) of Fact 3, we have

$$
\begin{aligned}
f_{i}(u . t, v . t) & =\operatorname{tr}_{K_{\xi . t} / k}\left(\varphi_{t, i}(u) \varphi_{t, i}(v)^{\tau^{\prime}}\right) \\
& =\operatorname{tr}_{K_{\xi . t} / k}\left(\phi_{\xi, t}(u) \phi_{\xi^{*}, t}(v)^{\tau^{\prime}}\right) \\
& =\operatorname{tr}_{K_{\xi . t} / k}\left(\phi_{\xi, t}\left(u v^{\tau}\right)\right) \\
& =\operatorname{tr}_{K_{\xi} / k}\left(u v^{\tau}\right)=f_{i}(u, v) .
\end{aligned}
$$

Here we use in the third line that $\tau^{\prime} \circ \phi_{\xi^{*}, t}=\phi_{\xi, t} \circ \tau$ since both induce $\sigma$ on the ground field $k$ and

$$
\begin{aligned}
\phi_{\xi^{*}, t}\left(\lambda_{\xi^{*}}\right)^{\tau^{\prime}} & =\left(t \lambda_{\xi^{*} . t}\right)^{\tau^{\prime}}=t^{\sigma} \lambda_{\xi . t}^{-1}=\left(t \lambda_{\xi . t}\right)^{-1} \\
& =\phi_{\xi, t}\left(\lambda_{\xi}\right)^{-1}=\phi_{\xi, t}\left(\lambda_{\xi}^{-1}\right)=\phi_{\xi, t}\left(\lambda_{\xi^{*}}^{\tau}\right) .
\end{aligned}
$$

In the orthogonal case for $p=2$, the forms $Q_{i}$ are preserved by a similar argument.

In case (i) (b) of Fact 3, let $\delta \in K_{\xi}$ be the element used there, and let $\delta^{\prime} \in K_{\xi . t}$ be the analogous element in the $\xi . t$-block. We may assume $\delta=\delta^{\prime}=1$ when $\varepsilon=1$. Then the same computation as in the previous case verifies that $\varphi_{t}$ preserves the forms $f_{i}$ (and $Q_{i}$ ). Now assume that $\varepsilon=-1$. If $t=1$, there is nothing to show, so $t=-1$. When $\chi \neq \chi$.t, then $\xi \neq \xi$.t for $\xi \in \bar{\chi}$, so we can assume that $\delta^{\prime}=\phi_{\xi, t}(\delta)$. Then

$$
\begin{aligned}
f_{i}(u . t, v . t) & =\operatorname{tr}_{K_{\xi . t} / k}\left(\delta^{\prime} \varphi_{t, i}(u) \varphi_{t, i}(v)^{\tau^{\prime}}\right) \\
& =\operatorname{tr}_{K_{\xi . t} / k}\left(\delta^{\prime} \phi_{\xi, t}(u) \phi_{\xi, t}(v)^{\tau^{\prime}}\right) \\
& =\operatorname{tr}_{K_{\xi . t} / k}\left(\delta^{\prime} \phi_{\xi, t}\left(u v^{\tau}\right)\right)
\end{aligned}
$$




$$
\begin{aligned}
& =\operatorname{tr}_{K_{\xi, t} / k}\left(\phi_{\xi, t}(\delta) \phi_{\xi, t}\left(u v^{\tau}\right)\right) \\
& =\operatorname{tr}_{K_{\xi} / k}\left(\delta u v^{\tau}\right)=f_{i}(u, v) .
\end{aligned}
$$

So assume $\chi=\chi$.t so that $\xi=\xi$.t (for $\xi \in \bar{\chi}$ ), $\tau=\tau^{\prime}$, and $\delta^{\prime}=\delta$. Then

$$
\begin{aligned}
f_{i}(u . t, v . t) & =\operatorname{tr}_{K_{\xi . t} / k}\left(\delta^{\prime} \varphi_{t, i}(u) \varphi_{t, i}(v)^{\tau^{\prime}}\right) \\
& =\operatorname{tr}_{K_{\xi / k}}\left(\delta \zeta \phi_{\xi, t}(u) \zeta^{\tau^{\prime}} \phi_{\xi, t}(v)^{\tau^{\prime}}\right) \\
& =\operatorname{tr}_{K_{\xi / k}}\left(\zeta^{q^{k / 2}+1} \delta \phi_{\xi, t}\left(u v^{\tau}\right)\right) \\
& =\operatorname{tr}_{K_{\xi / k}}\left(\phi_{\xi, t}\left(\delta u v^{\tau}\right)\right) \\
& =\operatorname{tr}_{K_{\xi / k}}\left(\delta u v^{\tau}\right)=f_{i}(u, v) .
\end{aligned}
$$

Here we use in the third line that $\zeta^{q^{k \chi / 2}+1}=-1$ and $\phi_{\xi, t}(\delta)=-\delta$ since $\phi_{\xi, t}=\tau$ is the unique involution $x \mapsto x^{q^{k / 2}}$ in this case.

Assume now we are in case (ii). Then we may again assume that $t=-1$ and $p>2$ since $|T| \leq|Z| \leq 2$, and also $\chi=X \pm 1 \neq \chi . t=X \mp 1$. Then we have $\varphi_{\xi, t}=\phi_{\xi, t}=\mathrm{id}_{k}$ so that $f_{i}$ is preserved according to Fact 3 . So we have shown that $\varphi_{t}$ preserves the forms $f_{i}$ (and $Q_{i}$ ).

Now let us fix $h \in \mathbf{C}_{\text {conf }}^{2}(g)$. We want to understand the shape of $h$.

Step 1: h stabilizes each $V_{\chi}(g)(\chi \in k[X]$ irreducible). Assume that

$$
h \in \mathbf{C}_{\text {conf }}^{2}\left(\left.g\right|_{V}\right) \leq \mathbf{C}_{\text {conf }}\left(\left.g\right|_{V}\right)
$$

does not stabilize each subspace $V_{\xi}(g)$ of $V(\xi \in \bar{\chi})$. Write $\bar{\chi}=\left\{\xi_{1}, \ldots, \xi_{l}\right\}$ and assume that $V_{\xi_{1}}(g) . h=V_{\xi_{2}}(g)$. Take

$$
f=\left(M_{1}, M_{2}, *, \ldots, *\right) \in \mathbf{C}\left(\left.g\right|_{V}\right) \leq \mathbf{C}_{\text {conf }}\left(\left.g\right|_{V}\right),
$$

where the $j$ th component of $f$ acts on $V_{\xi_{j}}(g)(j=1, \ldots, l)$; then

$$
f^{h}=h^{-1} f h=\left(*, M_{1}^{h}, *, \ldots, *\right) .
$$

Now there are three cases according to the classification presented in Fact 3. If $G=\mathrm{GL} u(q)$, we can take $M_{2}=1_{V_{\xi_{2}}(g)}$ and $M_{1}$ far away from $k^{\times} \operatorname{id}_{V_{\xi_{1}}(g)}$. Then $[f, h]=\left(*, M_{1}^{h}, *, \ldots, *\right)$ is far away from $k^{\times} \operatorname{id}_{V}$. If $G$ is one of $\operatorname{Sp}_{u}(q)$, $\mathrm{GO}_{u}(q)$, or $\operatorname{GU} u(q), \xi_{1}$ is not self-dual and $\xi_{2} \neq \xi_{1}^{*}$, we can do the same as before. When $\xi_{1}^{*}=\xi_{2}$ in this case, we must have $M_{2}=\left(M_{1}^{-\sigma}\right)^{\top}$ so that

$$
[f, h]=\left(*, M_{1}^{\sigma \top} M_{1}^{h}, *, \ldots, *\right) .
$$

Again we can choose $M_{1} \in \prod u \mathrm{GL}_{c_{i \xi_{1}}}\left(K_{\xi_{1}}\right)$ such that $\left(M_{1}^{\sigma}\right)^{\top} M_{1}^{h}$ is far away from $Z$. In the last case, $\xi_{1}=\xi_{1}^{*}$ is self-dual. Then again $M_{1}$ and $M_{2}$ are independent of each other, and we can choose $M_{2}=1_{V_{\xi_{2}}}(g)$. The only restriction 
on $M_{1}$ is that it lies in $\prod_{u} \mathrm{GU}_{c_{i \xi_{1}}}\left(K_{\xi_{1}}\right)$ if $\xi_{1} \neq X \pm 1$ or $G$ is $\mathrm{GU}_{u}(q)$ (see Fact 3 (i) (b)) resp. $M_{1} \in \prod_{u} X_{c_{i \xi_{1}}}(k)$ in the opposite case when $\xi_{1}=X \pm 1$, where $G=X_{U}(q)(X=\mathrm{Sp}$ or GO; see Fact 3 (ii)), so again we can choose $M_{1}$ such that $[f, h]=\left(*, M_{1}^{h}, *, \ldots, *\right)$ is far away from $Z$. In all cases, we get a contradiction. This shows that $h \in \mathbf{C}_{\text {conf }}^{2}(g)$ fixes each $V_{\chi}(g) \in \mathcal{V}(\chi \in k[X]$ irreducible).

Assume now that $\left.h\right|_{V_{\chi}(g)}=M . \alpha$, where $\alpha$ corresponds to an element of

$$
T^{l_{\chi}}=\left\{s^{l_{\chi}} \mid s \in T\right\}
$$

which induces a non-trivial field automorphism on $K_{\chi}$ and $M$ is an ultraproduct of matrices over $K_{\chi}$. This is the case since $h \in \mathbf{C}_{\text {conf }}(g)$ and by the above definition of $\varphi_{t}$.

Step 2: the automorphism $\alpha$ equals the identity $\operatorname{id}_{K_{\chi}}$. Then, for $\lambda \in K_{\chi}$, we have $(\lambda \text { id })^{h}=\lambda^{\alpha}$ id $=\left(\lambda^{\alpha} \lambda^{-1}\right) \lambda$ id. This implies that, for all $\lambda \in K_{\chi}^{\times}$stabilizing the forms $f_{i}$ (or $Q_{i} ; i \in I$ ) on $V_{\chi}\left(g\right.$ ), we have $\lambda^{\alpha} \lambda^{-1} \in Z \leq k^{\times}$. When $G=\operatorname{GL} u(q)$ or $\chi$ is not self-dual, there is no restriction on $\lambda$ (of course, if $G$ is one of $\mathrm{Sp}_{u}(q), \mathrm{GO}_{u}(q)$, or $\mathrm{GU}_{u}(q)$, then if $h$ acts as $M$ on $V_{\chi}(g)$, it must act as $\left(M^{-\sigma}\right)^{\top}$ on $\left.V_{\chi^{*}}(g)\right)$. Hence, in this case, for each $\lambda^{\times} \in K_{\chi}$, there exists $\kappa_{\lambda} \in k^{\times}$ such that $\lambda^{\alpha} \lambda^{-1}=\kappa_{\lambda}$. However, then every vector $\lambda \in K_{\chi}$ is an eigenvector of the $k$-linear map $\alpha$, which forces $\alpha=\mathrm{id}_{K_{\chi}}$, since $1 \in K_{\chi}$ is fixed, a contradiction.

In the opposite case, $G$ is one of $\operatorname{Sp}_{u}(q), \mathrm{GO}_{u}(q)$, or $\mathrm{GU}_{u}(q)$ and $\chi$ is selfdual. Then we are in case (i) (b) and (ii) of Fact 3. Recall that $\tau: K_{\chi} \rightarrow K_{\chi}$ is defined by $\left.\tau\right|_{k}=\sigma$ and $\tau: \lambda_{\chi} \mapsto \lambda_{\chi}^{-1}$, where $\lambda_{\chi} \in K_{\chi}$ is the root of $\chi$. Then $\tau^{2}=\mathrm{id}_{K_{\chi}}$ and $\tau=\mathrm{id}_{K_{\chi}}$ if and only if we are in case (ii) of Fact 3. Here, if we are in case (i) (b) of Fact 3, $\left.\mathbf{C}(g)\right|_{V_{\chi}(g)}$ is an ultraproduct of unitary groups over the field $K_{\chi}$ equipped with the involution $\tau$. In case (ii) of that fact, $\left.\mathbf{C}(g)\right|_{V_{\chi}(g)}$ is an ultraproduct of symplectic resp. orthogonal groups over $K_{\chi}=k=\mathbb{F}_{q}$. We proceed as follows. Find totally singular $K_{\chi}$-subspaces

$$
U={\overline{\left(U_{i}\right)}}_{i \in I}, U^{\prime}={\overline{\left(U_{i}^{\prime}\right)}}_{i \in I}, U^{\prime \prime}={\overline{\left(U_{i}^{\prime \prime}\right)}}_{i \in I} \in \mathcal{V} \quad \text { of } V_{\chi}(g)
$$

(in the sense of Remark 8) such that $U \oplus U^{\prime}=U \oplus U^{\prime \prime}=V_{\chi}(g), U^{\prime} \cap U^{\prime \prime}=0$, and $\operatorname{dim}(U)=\operatorname{dim}\left(U^{\prime}\right)=\operatorname{dim}\left(U^{\prime \prime}\right)=\operatorname{dim}\left(V_{\chi}(g)\right) / 2$. We may assume that

$$
\operatorname{dim}_{K_{\chi}}\left(U_{i}\right)=\operatorname{dim}_{K_{\chi}}\left(U_{i}^{\prime}\right)=\operatorname{dim}_{K_{\chi}}\left(U_{i}^{\prime \prime}\right)
$$

and that the restrictions $\left.f_{i}\right|_{U_{i} \times U_{i}^{\prime}}$ and $\left.f_{i}\right|_{U_{i} \times U_{i}^{\prime \prime}}$ are non-degenerate $(i \in \underline{I \text {, as we }}$ may by modifying $U_{i}, U_{i}^{\prime}$, and $U_{i}^{\prime \prime}$ a little if necessary). Then define $f^{\prime}={\overline{\left(f_{i}^{\prime}\right)_{i \in I}}}_{\text {, }}$,

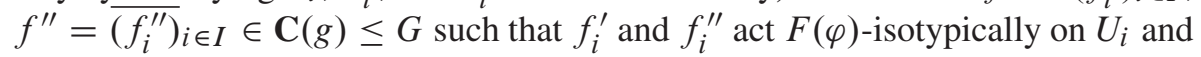
such that $f_{i}^{\prime}$ resp. $f_{i}^{\prime \prime}$ act $F\left(\varphi^{*}\right)$-isotypically on $U_{i}^{\prime}$ resp. $U_{i}^{\prime \prime}(i \in I)$ for a fixed 
irreducible polynomial $\varphi \in K_{\chi}[X]$ which is not self-dual with respect to $\tau$. Then $\left.f^{\prime h}\right|_{V_{\chi}(g)}=\left.z^{\prime} f^{\prime}\right|_{V_{\chi}(g)}$ and $\left.f^{\prime \prime h}\right|_{V_{\chi}(g)}=\left.z^{\prime \prime} f^{\prime \prime}\right|_{V_{\chi}(g)}$ for $z^{\prime}, z^{\prime \prime} \in Z$. Note that

$$
\begin{gathered}
q_{\varphi \cdot z^{\prime-1}}\left(\left.z^{\prime} f^{\prime}\right|_{V_{\chi}(g)}\right)=q_{\varphi}\left(\left.f^{\prime}\right|_{V_{\chi}(g)}\right)=1 / 2, \\
q_{\varphi . z^{\prime \prime-1}}\left(\left.z^{\prime \prime} f^{\prime \prime}\right|_{V_{\chi}(g)}\right)=q_{\varphi}\left(\left.f^{\prime \prime}\right|_{V_{\chi}(g)}\right)=1 / 2,
\end{gathered}
$$

and $\varphi \cdot z^{\prime-1}$ and $\varphi \cdot z^{\prime \prime-1}$ are both also not self-dual since $\varphi \in K_{\chi}[X]$ is not self-dual and $z^{\prime-1}, z^{\prime \prime-1} \in Z$ so that $z^{\prime-\tau}=z^{\prime-\sigma}=z^{\prime}$ and $z^{\prime \prime-\tau}=z^{\prime \prime}-\sigma=z^{\prime \prime}$, whence, e.g., $\left(\varphi \cdot z^{\prime-1}\right)^{*}=\varphi^{*} \cdot z^{\prime-1} \neq \varphi \cdot z^{\prime-1}$. Then $h$ must stabilize the decompositions $V_{\chi}(g)=U \oplus U^{\prime}=U \oplus U^{\prime \prime}$, so it must stabilize $U$. But on the $h$-invariant totally isotropic subspace $U$, we can do the same argument as above for $G=\operatorname{GL} u(q)$ to see that $\alpha=\operatorname{id}_{K_{\chi}}$. Hence we have obtained that $\left.h\right|_{V_{\chi}(g)}=M \in \prod u \mathbf{M}_{c_{i \chi}}\left(K_{\chi}\right)$ so that $h \in \mathbf{C}(g)$.

Step 3: we have that $\left.h\right|_{V_{\chi}(g)}=M=\lambda \operatorname{id}$ for $\lambda \in K_{\chi}$. According to Fact 3, we can find $V_{i}(i \in I)$ such that $\left(V_{i}\right)_{i \in I}=V_{\chi}(g)$ such that either all $V_{i}$ are totally singular (case (i) (a) of that fact, i.e., if $\chi$ is not self-dual; this includes the case $G=\mathrm{GL}_{u}(q)$ ) or $H_{i}$ preserves a unitary form (case (i) (b) of Fact 3 ) or a symplectic or orthogonal form (and a corresponding quadratic form when $p=2$, case (ii) of Fact 3) over $K_{\chi}$ on $V_{i}(i \in I)$. Note that it follows from the classification in $[4$, Subsection 3.4.2, §1] that orthogonally indecomposable blocks involving a Frobenius block of size $\geq 2$ are non-central in the ambient projective linear classical group. This shows that $q_{\xi}(M)=0$ for all $\xi \in K_{\chi}[X]$ of degree $\geq 2$. Assume now that there exist distinct $\lambda, \mu \in K_{\chi}^{\times}$such that $q_{X-\lambda}(M), q_{X-\mu}(M) \geq \varepsilon>0$. If $G=\mathrm{GL} u(q)$ or we are in case (i) (a) of Fact 3,

$$
F(X-\lambda) \oplus F(X-\mu)=\operatorname{diag}(\lambda, \mu) \in \mathrm{GL}_{2}\left(K_{\chi}\right)
$$

is mapped to a non-central element in $\mathrm{PGL}_{2}\left(K_{\chi}\right)$ so that, by the assumption, since we have "many" of these blocks, $\left.h\right|_{V_{\chi}(g)}$ would not commute modulo scalars with all of $\left.\mathbf{C}(g)\right|_{V_{\chi}(g)} \cong \prod u \mathbf{M}_{c_{i \chi}}\left(K_{\chi}\right)$. In case (i) (b) of Fact 3, we use the same argument for a block of shape $\operatorname{diag}\left(\lambda, \lambda^{-\tau}, \mu, \mu^{-\tau}\right)$ acting on a four-dimensional $\left(K_{\chi}, \tau\right)$-unitary space. In case (ii) of Fact 3 , we use the same argument with a block $\operatorname{diag}\left(\lambda, \lambda^{-1}, \mu, \mu^{-1}\right)$ acting on a four-dimensional symplectic or orthogonal space. In total, we get that $M=\lambda$ id for $\lambda \in K_{\chi}$. If we are in case (i) (b) of Fact 3, we have the additional assumption that $\mathrm{N}_{\tau}(\lambda)=1$, where $\mathrm{N}_{\tau}: K_{\chi} \rightarrow\left(K_{\chi}\right)_{\tau}$ is the field norm $x \mapsto x x^{\tau}$. In case (ii) of Fact 3, we must have $\lambda^{2}=1$.

Step 4: the precise shape of $C:=\mathbf{C}_{\text {conf }}^{2}(g)$. We know now $\left.h\right|_{V_{\chi}(g)}=\lambda_{\chi}(h)$ id for each irreducible $\chi \in k[X]$, and so $h$ commutes with all of $\mathbf{C}(g)$. In order that $h \in \mathbf{C}_{\text {conf }}^{2}(g)$, we still need to check that $\left[h, \varphi_{t}\right]=z$ id, where $t \in T$ is the generator fixed above and $z \in Z$. Let $\chi \in k[X]$ run through a system of representatives 
of the orbits of the action of $T$ and $\bullet *$ on the irreducible polynomials (the action of $\bullet *$ is only used when $G$ is not GLu$(q)$ ). This means $z h=h^{\varphi_{t}}$, so since $\left.h\right|_{V_{\chi}(g)}=\lambda_{\chi}(h)$ id, we must have $\left.h\right|_{V_{\chi . t}(g)}=z^{-1} \varphi_{\chi, t}\left(\lambda_{\chi}(h)\right) \operatorname{id}_{V_{\chi . t}}$ so that $h$ is determined on all of $V=V_{\bar{\chi}}(g)$ by $\lambda_{\chi}(h)$. In this situation, the only condition that needs to be satisfied is that

$$
\left.h\right|_{V_{\chi}(g)}=\left.h\right|_{V_{\chi . t} l_{\chi}(g)}=\lambda_{\chi}(h) \mathrm{id}=z^{-l_{\chi}} \varphi_{\chi, t}^{l_{\chi}}\left(\lambda_{\chi}(h)\right) \mathrm{id} .
$$

Note that

$$
\varphi_{\chi, t}^{l_{\chi}}: K_{\chi} \cong \mathbb{F}_{q^{d k_{\chi}}} \rightarrow K_{\chi} \cong \mathbb{F}_{q^{d k_{\chi}}} \quad(d=1,2)
$$

is given by $x \mapsto \kappa_{\chi} x^{q^{d k_{\chi} / m_{\chi}}}$, where $\kappa_{\chi}=1$ unless $p>2, \varepsilon=-1, t=-1$, and $\chi . t=\chi$, in which case $\kappa_{\chi}=\zeta$ is the scalar chosen above (which depends on $\chi$ ), so that the previous condition becomes

$$
z^{l_{\chi}}=\kappa_{\chi}\left(\lambda_{\chi}(h)\right)^{q^{d k_{\chi} / m_{\chi}-1}} .
$$

Hence we can write $C$ as follows. When $G=\operatorname{GL} u(q)$, we have

$$
C=\left\{\begin{array}{l|l}
\left.h=\bigoplus_{\substack{\chi \text { irreducible } \\
X^{o} \equiv \mu(\chi) \\
q_{\chi}(g)>0}} \lambda_{\chi}(h) \operatorname{id}_{V_{\chi}(g)} \mid \begin{array}{l}
\text { there exists } z \in Z \text { such that } \\
\lambda_{\chi . t}(h)=z^{-1} \varphi_{\chi, t}\left(\lambda_{\chi}(h)\right) \text { for all } \chi
\end{array}\right\} .
\end{array}\right.
$$

Here the condition from equation (8.3) is equivalent to equation (8.2) for $\chi$ running through a system of representatives of the action of $T$ on the set

$$
P:=\left\{\chi \in k[X] \text { irreducible } \mid \chi \text { divides } X^{o}-\mu \text { and } q_{\chi}(g)>0\right\} .
$$

For $G$ one of $\operatorname{Sp}_{u}(q), \mathrm{GO}_{u}(q)$, or $\mathrm{GU}_{u}(q)$, we have

$$
C=\left\{\bigoplus_{\substack{\chi \text { irreducible } \\ X^{o} \equiv \mu(\chi) \\ q_{\chi}(g)>0}} \lambda_{\chi}(h) \operatorname{id}_{V_{\chi}(g)} \mid R\right\}
$$

where condition $R$ is that there exists $z \in Z$ such that $\lambda_{\chi . t}(h)=z^{-1} \varphi_{\chi, t}\left(\lambda_{\chi}(h)\right)$ for all $\chi \in P$ (as in the previous case) and $\lambda_{\chi}(h) \lambda_{\chi^{*}}(h)^{\tau}=1$ for all $\chi \in P$, where $\tau: K_{\chi^{*}} \rightarrow K_{\chi}$ is defined as in Fact 3. If $G$ is one of $\operatorname{Sp}_{u}(q)$ or $\operatorname{GO}_{u}(q)$ and $\chi=\chi^{*} \neq X \pm 1$ is self-dual, this means $k_{\chi}$ is even and $\lambda_{\chi}(h)^{q^{k_{\chi} / 2}+1}=1$. Also, in this case, if $\chi=X \pm 1$, it means $\lambda_{\chi}(h)^{2}=1$. If $G=\operatorname{GU} u(q)$ and $\chi=\chi^{*}$, this means that $k_{\chi}$ is odd (since $\tau$ needs to induce $\sigma: x \mapsto x^{q}$ on $k=\mathbb{F}_{q^{2}}$ ) and $\lambda_{\chi}(h)^{q^{k \chi}+1}=1$. 


\section{Distinction of metric ultraproducts}

Now we want to distinguish all (simple) metric ultraproducts $\bar{G}=\bar{X} u(q)$ for distinct pairs $(X, q)$, where $X \in\{\mathrm{GL}, \mathrm{Sp}, \mathrm{GO}, \mathrm{GU}\}$ and $q$ is a prime power (all but $\operatorname{PSp}_{u_{1}}(q)$ and $\mathrm{PGO}_{u_{2}}(q)$ as mentioned in Theorem 1). For a group $H$, define the quantity

$$
e_{H}(o):=\max _{h \in H: h^{o}=1_{H}} \exp \left(\mathbf{C}^{2}(h)\right) .
$$

Clearly, when $H \cong L$, we have

$$
e_{H}(o)=e_{L}(o) \quad \text { for all values } o \in \mathbb{Z}_{+} .
$$

Our strategy is to compute $e_{H}(o)$ for the groups $H=\bar{G}$, where $\bar{G}=\bar{X} u(q)$ as above, for certain values of $o$ to distinguish these groups (with the only exception $\operatorname{PSp}_{u_{1}}(q) \cong \operatorname{PGO}_{u_{2}}(q)$ ?).

\subsection{Computation of $e_{\bar{G}}(o)$ when $\operatorname{gcd}\{o, p\}=\operatorname{gcd}\{o,|Z|\}=1$}

If $o$ is coprime to $|Z|$ (and by semisimplicity of $g \in G$ coprime to $p$ ), from the previous considerations, we can compute $e \bar{G}(o)$. Note that, in this situation, when $g^{o}=\mu \in Z$, we can replace $g$ by $g^{\prime}=\lambda g \in G$ such that $g^{\prime o}=1$, choosing $\lambda \in Z$ such that $\lambda^{o}=\mu^{-1}$, since the homomorphism $Z \rightarrow Z, x \mapsto x^{o}$ is then bijective. So we may assume that $g^{o}=1$. Then

$$
P \subseteq Q:=\left\{\chi \in k[X] \text { irreducible } \mid \chi \text { divides } X^{o}-1\right\} .
$$

The case $G=\operatorname{GL} u(q)$. From equation (8.3), we see that the bigger the group $T$ is, for an element $h \in \mathbf{C}_{\text {conf }}^{2}(g)$, the more restrictions are imposed to the scalars $\lambda_{\chi}(h) \in K_{\chi}^{\times}(\chi \in P)$. Also the bigger the set $P$ is, the "bigger" is the group $\mathbf{C}_{\text {conf }}^{2}(g)$, i.e., there are more components. Hence, to optimize the exponent of $\mathbf{C}^{2}(\bar{g})=\mathbf{C}_{\text {conf }}^{2}(g) / Z$, we choose $g$ such that

$$
P=Q \quad \text { and } \quad 0<q_{X-1}(g) \neq q_{\chi}(g)>0 \quad \text { for all } \chi \in P \backslash\{X-1\} .
$$

Namely, then $T=\operatorname{stab}_{Z}(q(g))$ must fix the polynomial $X-1$ so that we must have $T=\mathbf{1}$. Set

$$
f_{q}(o):=\min \left\{q^{e}-1 \mid o \text { divides } q^{e}-1\right\} .
$$

Equation (8.3) then gives

$$
e_{\bar{G}}(o)=\exp \left(\mathbf{C}^{2}(\bar{g})\right)=\exp \left(\mathbf{C}_{\mathrm{conf}}^{2}(g) / Z\right)= \begin{cases}1 & \text { if } o=1 \\ f_{q}(o) & \text { if } o>1\end{cases}
$$


Let us demonstrate equation (9.1). The first equality in it holds by the previous argument. When $o=1$, we have $\bar{g}=1_{\bar{G}}$, and so

$$
\mathbf{C}^{2}(\bar{g})=\mathbf{Z}(\bar{G})=\mathbf{1}
$$

so that $e_{\bar{G}}(1)=1$. Now assume $o>1$. For each $\chi \in P$, if $\lambda \in \bar{k}^{\times}$is a root of $\chi$, the condition $\chi \mid X^{o}-1$ is equivalent to $\lambda^{o}=1$. Also $K_{\chi}=k[\lambda]$. Let $\mu \in \bar{k}^{\times}$be an element of order $o$ with minimal polynomial $\xi \in k[X]$. Then, if $\lambda$ is a root of $\chi \in P$, we must have $\lambda^{o}=1$ and thus $\lambda=\mu^{f}$ for some $f \in \mathbb{N}$. Hence

$$
K_{\chi}=k[\lambda]=k\left[\mu^{f}\right] \subseteq k[\mu]=K_{\xi}
$$

so that, in equation (8.3), $\operatorname{ord}\left(\lambda_{\chi}(h)\right)$ divides $\left|K_{\chi}^{\times}\right|$which divides $\left|K_{\xi}^{\times}\right|=f_{q}(o)$. This shows that

$$
\begin{array}{r}
\exp \left(\mathbf{C}^{2}(\bar{g})\right)\left|\exp \left(\mathbf{C}_{\text {conf }}^{2}(g)\right)\right| \operatorname{lcm}\left\{\left|K_{\chi}^{\times}\right| \mid \chi \text { divides } X^{o}-1\right\} \\
=\left|K_{\xi}^{\times}\right|=\left|k[\mu]^{\times}\right|=f_{q}(o) .
\end{array}
$$

To show that $\exp \left(\mathbf{C}^{2}(\bar{g})\right)=f_{q}(o)$, take $h \in \mathbf{C}_{\text {conf }}^{2}(g)$ such that $\lambda_{X-1}(h)=1$ and $\lambda_{\xi}(h)$ has order $f_{q}(o)=\left|K_{\xi}^{\times}\right|$in $\bar{k}^{\times}$. Then, when $\bar{h}^{l}=1 \bar{G}$, we must have $h^{l} \in Z$. But $\lambda_{X-1}(h)^{l}=1$ so that, since $q_{X-1}(g)>0$, it follows that $h^{l}=1_{G}$. Then $\lambda_{\xi}(h)^{l}=1$ so that $\exp \left(\mathbf{C}^{2}(\bar{g})\right) \geq l \geq \operatorname{ord}\left(\lambda_{\xi}(h)\right)=f_{q}(o)$. This completes the proof.

The case $G=\operatorname{Sp}_{u}(q)$ or $\mathrm{GO}_{u}(q)$. As in the linear case, equation (8.4) shows that the optimal exponent of $\mathbf{C}^{2}(\bar{g})$ is obtained when

$$
P=Q \quad \text { and } \quad 0<q_{X-1}(g) \neq q_{\chi}(g)>0 \quad \text { for all } \chi \in P \backslash\{X-1\}
$$

so that $T=\mathbf{1}$. Set

$$
f_{q}^{\prime}(o):= \begin{cases}q^{e / 2}+1 & \text { if } f_{q}(o)=q^{e}-1, e \text { is even, and } o \mid q^{e / 2}+1, \\ f_{q}(o) & \text { otherwise. }\end{cases}
$$

Equation (8.4) then gives

$$
e_{\bar{G}}(o)=\exp \left(\mathbf{C}^{2}(\bar{g})\right)=\exp \left(\mathbf{C}_{\text {conf }}^{2}(g) / Z\right)= \begin{cases}1 & \text { if } o=1, \\ 2 & \text { if } o=2, \\ f_{q}^{\prime}(o) & \text { if } o>2\end{cases}
$$

We demonstrate equation (9.3). If $o=1$, we obtain, as in the linear case, that $\mathbf{C}^{2}(\bar{g})=\mathbf{1}$, and so $e_{\bar{G}}(1)=1$. If $o=2, g^{2}=1$, and so $P=\{X-1, X+1\}$. From equation (8.4), we see that, if $h \in \mathbf{C}_{\text {conf }}^{2}(g)$, we have

$$
\lambda_{X-1}(h)^{2}=\lambda_{X+1}(h)^{2}=1
$$


so that $h^{2}=1$. Also, defining $h$ by $\lambda_{X-1}(h):=1$ and $\lambda_{X+1}(h)=-1$, we obtain $h \notin Z$, so ord $\bar{G}(\bar{h})=e_{\bar{G}}(2)=2(1 \neq-1$ since the case $p=2$ does not occur due to the condition $\operatorname{gcd}\{o, p\}=1)$. Assume now that $o>2$. As in the linear case, for each $\chi \in P$, if $\lambda \in \bar{k}$ is a root of $\chi$, the condition that $\chi \mid X^{o}-1$ is equivalent to $\lambda^{o}-1$. Choose $\mu \in \bar{k}^{\times}$of order $o$, and let $\xi \in P$ be its minimal polynomial. Then, as previously, if $\lambda$ is a root of $\chi \in P$, we have $\lambda=\mu^{f}$ for some $f \in \mathbb{N}$. There are two cases.

In the first case, $\xi$ is not self-dual. This means that $\mu$ and $\mu^{-1}$ are not conjugate in $K_{\xi} / k$. If they were conjugate, say by an automorphism $\alpha$, i.e., $\mu^{\alpha}=\mu^{-1}$, then $\alpha=\tau \in \operatorname{Gal}\left(K_{\xi} / k_{k_{\xi}}\right)$ needs to be the unique involution (since $\mu \neq \mu^{-1}$ as $o>2$ ) given by $x \mapsto x^{q^{k_{\xi} / 2}}$; in particular, $e=k_{\xi}$ would need to be even. Hence this case is equivalent to either $e=k_{\xi}$ being odd or $\mu \mu^{\alpha}=\mu^{q^{k_{\xi} / 2}+1} \neq 1$, i.e., $o \nmid q^{e / 2}+1=q^{k_{\xi} / 2}+1$. This is precisely the opposite of the first case in equation (9.2). Here, for an element $h \in \mathbf{C}_{\text {conf }}^{2}(g)$, we can choose $\lambda_{\xi}(h) \in K_{\xi}^{\times}=k[\mu]^{\times}$ arbitrarily $\left(\lambda_{\xi^{*}}(h)\right.$ is then determined by $\left.\lambda_{\xi}(h)\right)$. Arguing as in the linear case, we obtain $\exp \left(\mathbf{C}^{2}(\bar{g})\right)=f_{q}(o)$. Indeed, for $h \in \mathbf{C}_{\text {conf }}^{2}(g)$, as above, $\operatorname{ord}\left(\lambda_{\chi}(h)\right)$ divides $f_{q}(o)$, and defining $h$ such that $\lambda_{X-1}(h)=1$ and $\lambda_{\xi}(h)$ has order $f_{q}(o)$, we see that $\operatorname{ord}_{\bar{G}}(\bar{h})=e_{\bar{G}}(o)=f_{q}(o)$.

In the opposite case, $\xi$ is self-dual and $\xi \neq X \pm 1$ as $o>2$. Then $e=k_{\xi}$ needs to be even and

$$
\mu \mu^{\tau}=\mu^{q^{k_{\xi} / 2}+1}=\lambda_{\xi}(h) \lambda_{\xi}(h)^{\tau}=\lambda_{\xi}(h)^{q^{k_{\xi} / 2}+1}=1,
$$

where $\tau$ is the involution $x \mapsto x^{q^{k_{\xi} / 2}}$ of $K_{\xi} \cong \mathbb{F}_{q^{k_{\xi}}}$ from Fact 3 (i) (b). This means that $o \mid q^{e / 2}+1$ and we are in the first case of equation (9.2). Note that, for each $\chi \in P$, the map $\tau$ restricts to an automorphism of each $K_{\chi} \subseteq K_{\xi}$ of order dividing two (as all the fields are finite). Then $\left.\tau\right|_{K_{\chi}}=$ id if and only if $k_{\chi} \mid k_{\xi} / 2$, and $\left.\tau\right|_{K_{\chi}}$ is the unique involution of $K_{\chi}$ if $k_{\xi} / k_{\chi}$ is odd. Now, if $\lambda \in \bar{k}^{\times}$is a root of $\chi$, then in the first case $\lambda^{2}=1$, and in the second case $\lambda^{q^{k} \chi / 2}+1=1$, so all $\chi \in P$ are self-dual. Hence, if $h \in \mathbf{C}_{\text {conf }}^{2}(g)$, for each $\chi \in P$, one of $\lambda_{\chi}(h)^{2}=1$ or $\lambda_{\chi}(h)^{q^{k_{\chi} / 2}+1}=1$ must hold. But $2 \mid q^{k_{\xi} / 2}+1$ if $p>2$, and in the second case, $q^{k_{\chi} / 2}+1 \mid q^{k_{\xi} / 2}+1$ since $k_{\xi} / k_{\chi}$ is then odd. Hence

$$
\exp \left(\mathbf{C}_{\text {conf }}^{2}(g)\right) \mid q^{e / 2}+1=q^{k_{\xi} / 2}+1=f_{q}^{\prime}(o) .
$$

Defining $h \in \mathbf{C}_{\text {conf }}^{2}(g)$ such that $\lambda_{X-1}(h)=1$ and $\lambda_{\xi}(h)$ has order $f_{q}^{\prime}(o)$, we see that $\operatorname{ord}_{\bar{G}}(\bar{h})=f_{q}^{\prime}(o)$.

The case $G=\mathrm{GU}_{u}(q)$. Here, as well, equation (8.4) shows that the optimal exponent of $\mathbf{C}^{2}(\bar{g})$ is obtained when $P=Q$ and $0<q_{X-1}(g) \neq q_{\chi}(g)>0$ for all $\chi \in P \backslash\{X-1\}$ so that $T=\mathbf{1}$. Set

$$
f_{q}^{\prime \prime}(o):= \begin{cases}q^{e}+1 & \text { if } f_{q^{2}}(o)=q^{2 e}-1, e \text { is odd, and } o \mid q^{e}+1, \\ f_{q^{2}}(o) & \text { otherwise. }\end{cases}
$$


Equation (8.4) gives

$$
e_{\bar{G}}(o)=\exp \left(\mathbf{C}^{2}(\bar{g})\right)=\exp \left(\mathbf{C}_{\mathrm{conf}}^{2}(g) / Z\right)= \begin{cases}1 & \text { if } o=1, \\ f_{q}^{\prime \prime}(o) & \text { if } o>1\end{cases}
$$

Again $e_{\bar{G}}(1)=1$ is clear. If $o>1$, take $\mu \in \bar{k}^{\times}$of order $o$ with minimal polynomial $\xi$. Then the argument proceeds as in the bilinear case. But the condition that $\xi$ is self-dual is here equivalent to $\mu$ being conjugate to $\mu^{-1}$ in $K_{\xi}$ by an automorphism $\tau$ such that $\left.\tau\right|_{k}=\sigma ; x \mapsto x^{q}$. This forces $e=k_{\xi}$ to be odd and $\mu^{q^{d k_{\xi} / 2}+1}=1$, i.e., $o \mid q^{e}+1=q^{k_{\xi}}+1$.

\subsection{Proof of Theorem 1}

Set $G_{j}:=X_{j} u_{j}\left(q_{j}\right), Z_{j}:=\mathbf{Z}\left(G_{j}\right)$, and $\bar{G}_{j}:=G_{j} / Z_{j}(j=1,2)$. Let $p_{j}$ be the characteristic of the field $\mathbb{F}_{q_{j}}(j=1,2)$. Assume that $\bar{G} \cong \bar{G}_{1} \cong \bar{G}_{2}$. We start by showing that $p_{1}=p_{2}$.

Determining the characteristic $p$. Choose $o$ large enough and coprime to $p_{1}$, $p_{2},\left|Z_{1}\right|,\left|Z_{2}\right|$. Then from equations (9.1), (9.3), and (9.4), we see that $e_{\bar{G}}(o)$ is of the form $q_{1}^{e_{1}} \pm 1$ and $q_{2}^{e_{2}} \pm 1$. If we have $e_{\bar{G}}(o)=q_{1}^{e_{1}}-1=q_{2}^{e_{2}}-1$ or $e_{\bar{G}}(o)=q_{1}^{e_{1}}+1=q_{2}^{e_{2}}+1$, then $q_{1}^{e_{1}}=q_{1}^{e_{2}}$ so that $p_{1}=p_{2}$ by the uniqueness of the prime factorization. So we may assume that $e_{\bar{G}}(o)=q^{e_{1}}-1=q_{2}^{e_{2}}+1$ for infinitely many $o$, and so for infinitely many pairs $\left(e_{1}, e_{2}\right) \in \mathbb{Z}_{+}^{2}$. If $p_{1} \neq p_{2}$, we get a contradiction to [1, Corollary 1.8]. Hence $p_{1}=p_{2}=: p$.

Determining $q^{d}$. We can now assume $q_{j}=p^{e_{j}}(j=1,2)$. Choose $j \in\{1,2\}$, and set $X:=X_{j}, q:=q_{j}$, and $d:=d_{j}$. Consider the quantity

$$
f:=\operatorname{gcd}\left\{e_{\bar{G}}(o) \mid o \in O\right\},
$$

where

$$
O:=\left\{o \in \mathbb{Z}_{+} \mid 2<o \text { coprime to } p,\left|Z_{1}\right|,\left|Z_{2}\right| ; e \bar{G}(o) \equiv-1 \text { modulo } p^{3}\right\}
$$

From equations (9.1), (9.3), and (9.4), it follows that, for every element $o \in O$, the number $e_{\bar{G}}(o)$ is either of the form $q^{d e}-1$ or $q^{e}+1$. But the second case is excluded by the condition that $e_{\bar{G}}(o) \equiv-1$ modulo $p^{3}$. Hence $q^{d}-1$ divides $e_{\bar{G}}(o)=q^{d e}-1$, and so $q^{d}-1$ divides $f$.

For a prime $r$, set $t_{r}:=\frac{q^{r}-1}{q-1}$. Then, for distinct primes $r$ and $s$, we have

$$
\begin{aligned}
\operatorname{gcd}\left\{t_{r}, t_{s}\right\} & =\operatorname{gcd}\left\{\frac{q^{r}-1}{q-1}, \frac{q^{s}-1}{q-1}\right\} \\
& =\frac{1}{q-1} \operatorname{gcd}\left\{q^{r}-1, q^{s}-1\right\}=\frac{q^{\operatorname{gcd}\{r, s\}}-1}{q-1}=1 .
\end{aligned}
$$


Hence the numbers $t_{r}$ ( $r$ prime), being pairwise coprime, have arbitrarily large prime divisors. Take for $r>2$ a prime such that $t_{r}$ has a prime divisor $o>p,\left|Z_{1}\right|$, $\left|Z_{2}\right|, q^{d}-1$. Then $o$ is coprime to $p,\left|Z_{1}\right|$, and $\left|Z_{2}\right|$ so that, by equations (9.1), (9.3), and (9.4), we have $e \bar{G}(o)\left|f_{q^{d}}(o)\right| q^{d r}-1$, as $o\left|q^{r}-1\right| q^{d r}-1$. Hence the number $f_{q^{d}}(o)$ must be one of $q^{d r}-1$ or $q^{d}-1$, the latter being excluded by the condition $o>q^{d}-1$, so $f_{q^{d}}(o)=q^{d r}-1$. If $X=\mathrm{GL}, X=\mathrm{Sp}$, or $X=\mathrm{GO}$, since $r$ is odd and $d=1$, equations (9.1) and (9.3) show that we must have $e_{\bar{G}}(o)=f_{q^{d}}(o)=q^{d r}-1=q^{r}-1 \equiv-1$ modulo $p^{3}$. Hence, in this case, we have $o \in O$. If $X=\mathrm{GU}$, it could be that $e_{\bar{G}}(o)=q^{r}+1$ when $o \mid q^{r}+1$. However,

$$
\operatorname{gcd}\left\{q^{r}+1, t_{r}\right\}\left|\operatorname{gcd}\left\{q^{r}+1, q^{r}-1\right\}\right| 2,
$$

and $t_{r}$ is always odd so that $\operatorname{gcd}\left\{q^{r}+1, t_{r}\right\}=1$, and hence also $\operatorname{gcd}\left\{q^{r}+1, o\right\}=1$ as $o \mid t_{r}$. This shows that here also $e_{\bar{G}}(o)=f_{q^{d}}(o)=q^{d r}-1=q^{2 r}-1 \equiv-1$ modulo $p^{3}$. Therefore, again $o \in O$.

Applying this argument for two different primes $r$, say $r_{1}$ and $r_{2}$, which produces two different primes $o$, say $o_{1}$ and $o_{2}$, we get

$$
\begin{aligned}
f & =\operatorname{gcd}\left\{e_{\bar{G}}(o) \mid o \in O\right\} \mid \operatorname{gcd}\left\{e_{\bar{G}}\left(o_{1}\right), e \bar{G}\left(o_{2}\right)\right\} \\
& =\operatorname{gcd}\left\{q^{d r_{1}}-1, q^{d r_{2}}-1\right\}=q^{\operatorname{gcd}\left\{d r_{1}, d r_{2}\right\}}-1=q^{d}-1 .
\end{aligned}
$$

Altogether, we have shown that $f=q^{d}-1$. Plugging in $j=1,2$, we obtain $q_{1}^{d_{1}}-1=q_{2}^{d_{2}}-1$ implying that $q_{1}^{d_{1}}=q_{2}^{d_{2}}$.

Now exclude all remaining possible isomorphisms but $\operatorname{PSp}_{u_{1}}(q) \cong \operatorname{PGO}_{u_{2}}(q)$.

Proof that $\mathrm{PGL}_{u_{1}}(q) \not \mathrm{PSp}_{u_{2}}(q)$ and $\mathrm{PGL}_{u_{1}}(q) \nRightarrow \mathrm{PGO}_{u_{2}}(q)$. Let

$$
G_{1}=\operatorname{GL}_{u_{1}}(q) \text { and } G_{2}=X_{u_{2}}(q) \text {, where } X=\mathrm{Sp} \text { or GO. }
$$

Set

$$
o:= \begin{cases}\frac{q^{2}+1}{2} & \text { if } p>2, \\ q^{2}+1 & \text { if } p=2 .\end{cases}
$$

Note that $o>2$ is coprime to $p,\left|Z_{1}\right|=q-1$, and $\left|Z_{2}\right|=|\{ \pm 1\}|$. Hence, by equation (9.3), we have $e_{\bar{G}}(o)=e_{\bar{G}_{2}}(o)=q^{2}+1$. Indeed, $o\left|q^{2}+1\right| q^{4}-1$. But $o \nmid q^{f}-1$ for $f$ properly dividing 4 since then $o \mid q^{2}-1$, but it is easy to see that $\operatorname{gcd}\left\{o, q^{2}-1\right\}=1$. This shows

$$
f_{q}(o)=q^{4}-1 \quad \text { and } \quad e_{\bar{G}}(o)=e_{\bar{G}_{2}}(o)=f_{q}^{\prime}(o)=q^{2}+1 .
$$

But then, by $\bar{G}_{1} \cong \bar{G}_{2}$, we obtain

$$
e_{\bar{G}}(o)=e_{\bar{G}_{1}}(o)=f_{q}(o)=q^{4}-1>q^{2}+1=e_{\bar{G}_{2}}(o)=e_{\bar{G}}(o),
$$

a contradiction. 
Proof that $\mathrm{PSp}_{u_{1}}\left(q^{2}\right) \not \operatorname{PGU}_{u_{2}}(q)$ and $\operatorname{PGO}_{u_{1}}\left(q^{2}\right) \not \operatorname{PGU}_{u_{2}}(q)$. Let

$$
G_{1}=X u_{1}\left(q^{2}\right) \text {, where } X=\mathrm{Sp} \text { or GO, and } G_{2}=\operatorname{GU}_{u_{2}}(q) \text {. }
$$

Define $o$ as in equation (9.5). Note that $o>2$ is coprime to $p,\left|Z_{1}\right|=|\{ \pm 1\}|$, and $\left|Z_{2}\right|=q+1$. Then, by equation (9.3), $e_{\bar{G}}(o)=e_{\bar{G}_{1}}(o)=f_{q^{2}}^{\prime}(o)=q^{2}+1$ (as above). But by equation (9.4), we obtain that +

$$
e \bar{G}=e_{\bar{G}_{2}}(o)=f_{q}^{\prime \prime}(o)=q^{4}-1>q^{2}+1=e_{\bar{G}_{1}}(o)=e \bar{G}(o)
$$

since $e=2$ is even, a contradiction. Set

Proof that $\mathrm{PGL}_{u_{1}}\left(q^{2}\right) \nRightarrow \operatorname{PGU}_{u_{2}}(q)$. Let $G_{1}=\operatorname{GL}_{u_{1}}\left(q^{2}\right), G_{2}=\operatorname{GU}_{u_{2}}(q)$.

$$
o:= \begin{cases}\frac{q^{5}+1}{5(q+1)} & \text { if } q \equiv-1 \text { modulo } 5, \\ \frac{q^{5}+1}{q+1} & \text { otherwise. }\end{cases}
$$

Note that $o$ is coprime to $p,\left|Z_{1}\right|=q^{2}-1$, and $\left|Z_{2}\right|=q+1 \mid q^{2}-1$. Indeed,

$$
\operatorname{gcd}\{o, q+1\}\left|\operatorname{gcd}\left\{\frac{q^{5}+1}{q+1}, q+1\right\}=\operatorname{gcd}\{5, q+1\}\right| 5 .
$$

But $5 \nmid o$ so that $\operatorname{gcd}\{o, q+1\}=1$. Similarly,

$$
\operatorname{gcd}\{o, q-1\}\left|\operatorname{gcd}\left\{q^{5}+1, q-1\right\}\right| \operatorname{gcd}\{2, q-1\} \mid 2 .
$$

But $o$ is always odd, $\operatorname{sog} \operatorname{gcd}\{o, q-1\}=1$. We have $o \mid q^{10}-1$ so that, from equation (9.1), we obtain that $e_{\bar{G}}(o)=e_{\bar{G}_{1}}(o)=f_{q^{2}}(o)$ is either $q^{10}-1$ or $q^{2}-1$. But clearly $q^{2}-1<o$ so that we must have $f_{q^{2}}(o)=q^{10}-1$. But equation (9.4) gives that

$$
e_{\bar{G}}(o)=e_{\bar{G}_{2}}(o)=f_{q}^{\prime \prime}(o)=q^{5}+1<q^{10}-1=f_{q}(o)=e_{\bar{G}_{1}}(o),
$$

a contradiction.

Remark 13. If $q_{i} \rightarrow u \infty$, then double centralizers of semisimple torsion elements are infinite groups.

Remark 14. If $q$ is even, then $\operatorname{PSp}_{u_{1}}(q) \cong \operatorname{PGO}_{u_{2}}(q)$ is possible due to the isomorphism $\mathrm{Sp}_{2 m}(q) \cong \mathrm{GO}_{2 m+1}(q)$. Also it seems hard to distinguish a group $\mathrm{PSp}_{u_{1}}(q)$ from a group $\mathrm{PGO}_{u_{2}}(q)$ for $q$ odd.

Acknowledgments. The author wants to thank Andreas Thom for interesting discussions about the topic. The content of this article is part of the author's PhD project [4]. 


\section{Bibliography}

[1] J.-H. Evertse, Linear forms in logarithms, preprint (2011), http://www.math . leidenuniv.nl/ ${ }^{\sim}$ evertse/dio2011-linforms.pdf.

[2] S. Gonshaw, M. Liebeck and E. O'Brien, Unipotent class representatives for finite classical groups, J. Group Theory 20 (2017), no. 3, 505-525.

[3] M. Liebeck and A. Shalev, Diameters of finite simple groups: Sharp bounds and applications, Ann. of Math. (2) 154 (2001), 383-406.

[4] J. Schneider, On ultraproducts of compact quasisimple groups, $\mathrm{PhD}$ thesis, TU Dresden, 2019; to appear on http://www . qucosa.de.

[5] A. Thom and J. Wilson, Metric ultraproducts of finite simple groups, Comp. Rend. Math. 352 (2014), no. 6, 463-466.

[6] A. Thom and J. Wilson, Some geometric properties of metric ultraproducts of finite simple groups, Israel J. Mathematics 227 (2018), no. 1, 113-129.

Received November 11, 2019; revised February 4, 2020.

\section{Author information}

Jakob Schneider, TU Dresden, 01062 Dresden, Germany.

E-mail: jakob.schneider@tu-dresden.de 